\title{
FUV and X-Ray Absorption in the Warm-Hot Intergalactic Medium
}

\author{
P. Richter · F.B.S. Paerels $\cdot$ J.S. Kaastra
}

Received: 20 September 2007 / Accepted: 21 September 2007 / Published online: 13 February 2008 (C) Springer Science+Business Media B.V. 2008

\begin{abstract}
The Warm-Hot Intergalactic Medium (WHIM) arises from shock-heated gas collapsing in large-scale filaments and probably harbours a substantial fraction of the baryons in the local Universe. Absorption-line measurements in the ultraviolet (UV) and in the Xray band currently represent the best method to study the WHIM at low redshifts. We here describe the physical properties of the WHIM and the concepts behind WHIM absorption line measurements of $\mathrm{HI}$ and high ions such as O VI, O VII, and O VIII in the far-ultraviolet and X-ray band. We review results of recent WHIM absorption line studies carried out with UV and X-ray satellites such as FUSE, HST, Chandra, and XMM-Newton and discuss their implications for our knowledge of the WHIM.
\end{abstract}

Keywords Galaxies: intergalactic medium - Quasars: absorption lines · Cosmology: large-scale structure of the Universe

\section{Introduction}

As recent cosmological simulations imply, the temperature of the intergalactic medium (IGM) undergoes a significant change from high to low redshifts parallel to the proceeding of large-scale structure formation in the Universe (e.g., Cen and Ostriker 1999; Davé et al. 2001). As a result, a substantial fraction of the baryonic matter in the local Universe is expected to reside in the so-called Warm-Hot Intergalactic Medium (WHIM). The WHIM represents a low-density $\left(n_{\mathrm{H}} \sim 10^{-6}-10^{-4} \mathrm{~cm}^{-3}\right)$, high-temperature $\left(T \sim 10^{5}-10^{7} \mathrm{~K}\right)$ plasma

\footnotetext{
P. Richter $(\bowtie)$

Institut für Physik, Universität Potsdam, Am Neuen Palais 10, 14469 Potsdam, Germany

e-mail: prichter@astro.physik.uni-potsdam.de

F.B.S. Paerels

Department of Astronomy and Columbia Astrophysics Laboratory, Columbia University, 550 West

120th Street, New York, NY 10027, USA
}

J.S. Kaastra

SRON, Sorbonnelaan 2, 3584 CA Utrecht, The Netherlands 
that primarily is made of protons, electrons, He II, and He III, together with traces of some highly-ionised heavy elements. The WHIM is believed to emerge from intergalactic gas that is shock-heated to high temperatures as the medium is collapsing under the action of gravity in large-scale filaments (e.g., Valageas et al. 2002). In this scenario, part of the warm (photoionised) intergalactic medium that gives rise to the Ly $\alpha$ forest in the spectra of distant quasars (QSO) is falling into the potential wells of the increasingly pronounced filaments, gains energy (through gravity), and is heated to high temperatures by shocks that run through the plasma.

Because of the low density and the high degree of ionisation, direct observations of the shock-heated and collisionally ionised WHIM are challenging with current instrumentation (in contrast to the photoionised IGM, which is easily observable through the Ly $\alpha$ forest). Diffuse emission from the WHIM plasma must have a very low surface brightness and its detection awaits UV and X-ray observatories more sensitive than currently available (see, e.g., Fang et al. 2005; Kawahara et al. 2006). The most promising approach to study the WHIM with observations at low redshift is to search for absorption features from the WHIM in FUV and in the X-ray regime in the spectra of quasars, active galactic nuclei (AGN) and other suited extragalactic background sources. As the WHIM represents a highly-ionised plasma, the most important WHIM absorption lines are those originating from the electronic transitions of high-ionisation state ions (hereafter referred to as "high ions") of abundant heavy elements such as oxygen and carbon. Among these, five-times ionised oxygen (O VI) is the most valuable high ion to trace the WHIM at temperatures of $T \sim 3 \times 10^{5} \mathrm{~K}$ in the FUV regime. In the X-ray band, the O VII and O VIII transitions represent the key observables to trace the WHIM at higher temperatures in the range $3 \times 10^{5}<T<10^{7}$. In addition to the spectral signatures of high ions of heavy elements the search for broad and shallow Ly $\alpha$ absorption from the tiny fraction of neutral hydrogen in the WHIM represents another possibility to identify and study the most massive WHIM filaments in the intergalactic medium with FUV absorption spectroscopy. Finally, for the interpretation of the observed WHIM absorption features in UV and X-ray spectra the comparison between real data and artificial spectra generated by numerical simulations that include realistic gas physics is of great importance to identify possible pitfalls related to technical and physical issues such as limited signal-to-noise ratios and spectral resolution, line-broadening mechanisms, non-equilibrium conditions, and others.

In this chapter, we review the physics and methodology of the UV and X-ray absorption measurements of warm-hot intergalactic gas at low redshift and summarise the results of recent observations obtained with space-based observatories. The outline of this chapter is the following. The ionisation conditions of the WHIM and the most important absorption signatures of this gas in the UV and X-ray band are presented in Sect. 2. Recent UV absorption measurements of the WHIM at low redshift are discussed in Sect. 3. Similarly, measurements of the WHIM in the X-ray are presented in Sect. 4. In Sect. 5 we compare the results from WHIM observations with predictions from numerical simulations and give an overview of WHIM measurements at high redshift. Finally, some concluding remarks are given in Sect. 6.

\section{Physical Properties of the WHIM}

\subsection{WHIM Ionisation Conditions}

The occurrence and characteristics of the WHIM absorption signatures in the FUV and Xray band are determined to a high degree by the ionisation conditions in the gas. We briefly 
discuss the WHIM ionisation properties, as this is crucial for interpretation of the WHIM absorption lines in FUV and X-ray spectra that arise in such warm-hot gas. Generally, there are two processes that determine the ionisation state of warm-hot gas in the intergalactic medium: collisional ionisation caused by the high temperature of the gas in collapsed structures and photoionisation by the cosmic FUV background.

\subsubsection{Hydrogen}

By far most of the mass of the WHIM is in the form of ionised hydrogen. Therefore, understanding the processes that lead to the ionisation of hydrogen is essential for the interpretation of WHIM absorption lines and for a reliable estimate of the baryon content of warm-hot intergalactic gas. The ionisation potential of neutral hydrogen is $13.6 \mathrm{eV}$ and thus both ionisation by particle collisions and ionisation by high-energy photons contribute to the ionisation of $\mathrm{H}$ I in warm-hot gas. We start with collisional ionisation, which is believed to dominate the ionisation of hydrogen at temperatures $>10^{5} \mathrm{~K}$.

In collisional ionisation equilibrium (CIE) - the most simple approach to characterise the ionisation conditions in low-density, high-temperature plasmas-the ionisation fraction depends only on the gas temperature. If we ignore any charge-exchange reactions (which is justified in case of hydrogen), the neutral hydrogen fraction in CIE is simply the ratio between the recombination coefficient $\alpha_{\mathrm{H}}(T)$ and the collisional ionisation coefficient $\beta_{\mathrm{H}}(T)$ :

$$
f_{\mathrm{H}, \text { coll }}=\frac{\alpha_{\mathrm{H}}(T)}{\beta_{\mathrm{H}}(T)} .
$$

Above gas temperatures of $\sim 1.5 \times 10^{4} \mathrm{~K}$ collisions by thermal electrons efficiently ionise hydrogen to a high degree, and already at $T \sim 3 \times 10^{4} \mathrm{~K}$ the neutral hydrogen fraction in the gas is less then one percent. For the temperature range that is characteristic for the WHIM, $T=10^{5}-10^{7} \mathrm{~K}$, one can approximate the ionisation fraction in a collisional ionisation equilibrium in the way

$$
\log f_{\mathrm{H}_{\mathrm{I}, \mathrm{coll}}} \approx 13.9-5.4 \log T+0.33(\log T)^{2} \text {. }
$$

where $T$ is in units K (Richter et al. 2006a; Sutherland and Dopita 1993). Thus, for WHIM gas with $T=10^{6} \mathrm{~K}$ the neutral hydrogen fraction in the gas in CIE is only $\sim 2.4 \times 10^{-7}$.

Next to particle collisions, photons with energies $>13.6 \mathrm{eV}$ contribute to the ionisation of the WHIM, in particular in the low-temperature WHIM tail at $\sim 10^{5} \mathrm{~K}$ and below. Such ionising photons in intergalactic space are indeed provided by the metagalactic ultraviolet (UV) background, originating from the hard radiation emitted by QSOs and AGN. Figure 1 shows the spectral shape of the UV background at $z=0$ (left panel) and the redshift-dependence of the hydrogen photoionisation rate from the UV background (right panel) based on the models by Haardt and Madau (1996).

Considering photoionisation, one generally can write for the neutral-hydrogen fraction in the gas:

$$
f_{\mathrm{H} \text {, photo }}=\frac{n_{\mathrm{e}} \alpha_{\mathrm{H}}(T)}{\Gamma_{\mathrm{H}_{\mathrm{I}}}},
$$

where $\alpha_{\mathrm{H}}(T)$ denotes the temperature-dependent recombination rate of hydrogen, $n_{\mathrm{e}}$ is the electron density, and $\Gamma_{\mathrm{HI}_{\mathrm{I}}}$ is the photoionisation rate. $\Gamma_{\mathrm{HI}}$ depends on the ambient ionising 

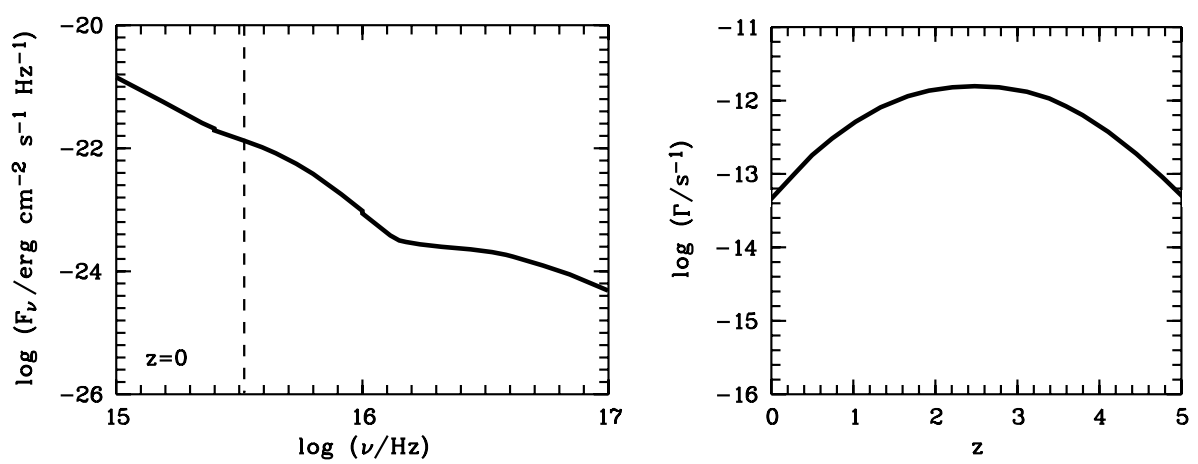

Fig. 1 Left panel: Spectral shape of the metagalactic UV background at $z=0$ (from Haardt and Madau 1996). Plotted is the flux of photons $\left(F_{v}=4 \pi J_{v}\right)$ against the frequency $v$. The hydrogen ionisation edge is indicated with a dashed line. Right panel: Redshift-dependence of the hydrogen photoionisation rate $\Gamma$ from the UV background for the range $z=0$ to $z=5$. Adapted from Haardt and Madau (1996)

radiation field $J_{v}$ (in units erg $\mathrm{cm}^{-2} \mathrm{~s}^{-1} \mathrm{~Hz}^{-1} \mathrm{sr}^{-1}$ ) in the WHIM provided by the metagalactic UV background (see Fig. 1):

$$
\Gamma_{\mathrm{H}}=4 \pi \int_{\nu_{\mathrm{L}}}^{\infty} \frac{\sigma_{\nu} J_{v}}{\mathrm{~h} v} \mathrm{~d} v \approx 2.5 \times 10^{-14} J_{-23} \mathrm{~s}^{-1} .
$$

Here, $v_{\mathrm{L}}$ is the frequency at the Lyman limit and $\sigma_{v}$ denotes the photoionisation cross section of hydrogen, which scales with $v^{-3}$ for frequencies larger than $v_{\mathrm{L}}$ (see Kaastra et al. 2008-Chap 9, this issue). We have introduced the dimensionless scaling factor $J_{-23}$ which gives the metagalactic UV radiation intensity at the Lyman limit in units $10^{-23}$ erg cm${ }^{-2} \mathrm{~s}^{-1} \mathrm{~Hz}^{-1} \mathrm{sr}^{-1}$. For $z=0$ we have $J_{-23} \sim 1-2$, while for $z=3$ the value for $J_{-23}$ is $\sim 80$, thus significantly higher (Haardt and Madau 1996). Assuming $n_{\mathrm{e}}=n_{\mathrm{H}}$ and inserting a proper function for $\alpha_{\mathrm{H}}(T)$, we finally can write for the logarithmic neutral hydrogen fraction in a purely photoionised WHIM plasma

$$
\log f_{\mathrm{H} \text {, photo }} \approx \log \left(\frac{16 n_{\mathrm{H}} T_{4}^{-0.76}}{J_{-23}}\right),
$$

where $n_{\mathrm{H}}$ is the hydrogen volume density in units $\mathrm{cm}^{-3}$ and $T_{4}$ is the temperature in units $10^{4} \mathrm{~K}$. Thus, for purely photoionised intergalactic gas at $z=0$ with $n=5 \times 10^{-6}$ and $T=10^{6} \mathrm{~K}$ we find that the neutral hydrogen fraction is $f_{\mathrm{H} \text { I,photo }} \sim 2.4 \times 10^{-6}$. This is ten times higher than for CIE, indicating that collisions dominate the ionisation fraction of hydrogen in intermediate and high-temperature WHIM regions. However, note that at lower temperatures near $T=10^{5} \mathrm{~K}$ at the same density we have $f_{\mathrm{H} \text { I,photo }} \sim f_{\mathrm{H} \text {, coll }}$. Since this is the WHIM temperature regime preferentially detected by UV absorption features (e.g., $\mathrm{O}$ VI and broad Ly $\alpha$ ), photoionisation is important and needs to be accounted for when it comes to the interpretation of WHIM absorbers observed in the FUV. From a WHIM simulation at $z=0$ including both collisional ionisation and photoionisation Richter et al. (2006b; see Fig. 2) find the following empirical relation between neutral hydrogen fraction and gas temperature for a WHIM density range between $\log n_{\mathrm{H}}=-5.3$ and -5.6 :

$$
\log f_{\mathrm{H}_{\mathrm{I}}} \approx 0.75-1.25 \log T .
$$


Fig. 2 The neutral hydrogen fraction, $\log f_{\mathrm{HI}}=\log \left(n_{\mathrm{HI}} / n_{\mathrm{H}}\right)$, in a WHIM simulation (photoionisation+collisional ionisation), is plotted as a function of the gas temperature, $\log T$. The light gray shaded indicates cells in the density range $\log n_{\mathrm{H}}=-5$ to -7 . The dark gray shaded area refers to cells that have $\log n_{\mathrm{H}}=-5.3$ to -5.6 , thus a density range that is characteristic for WHIM absorbers. Adapted from Richter et al. (2006b)

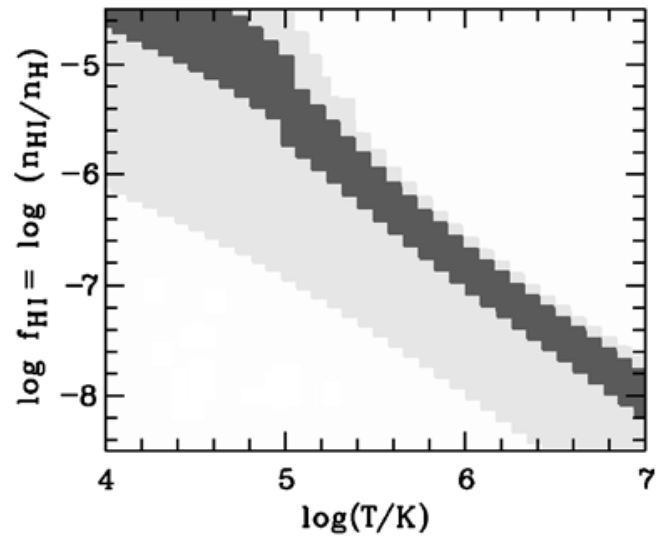

This equation may serve as a thumb rule to estimate ionisation fractions in WHIM absorbers at $z=0$ if the gas temperature is known (e.g., from measurements of the line widths; see Sect. 2.2.1)

\subsubsection{Oxygen and Other Metals}

While hydrogen provides most of the mass in the WHIM, the most important diagnostic lines to study this gas phase are from highly ionised metals such as oxygen, neon, carbon, magnesium, and others. Therefore, the understanding of the ionisation properties of the observed high ions of these elements is as important as for hydrogen. As for hydrogen, both collisional ionisation and photoionisation need to be considered. With its single electron, hydrogen can only be either neutral or fully ionised. Heavy elements, in contrast, have several electrons available and are-even at very high temperatures-usually only partly ionised. Thus, electronic transitions exist for such highly-ionised metals ("high ions") in warm-hot gas. Of particular importance for observations of the WHIM are the high ionisation states of oxygen, O VI, O VII, and O VIII, as they have strong electronic transitions in the UV (O VI) and at X-ray wavelengths (O VII \& O VIII) and oxygen is a relatively abundant element. Another important metal for observing warm-hot gas in the UV and Xray band is neon (Ne VII, Ne VIII, Ne IX, Ne X). In collisional ionisation equilibrium, the ionisation state of these elements is determined solely by the temperature of the gas. For each element, the ionisation fractions of the ionisation states (e.g., four-times vs. five-times ionised) then are characterised by the respective ionisation potentials (IPs) of the individual ionisation levels. For instance, at $T \sim 1-3 \times 10^{5} \mathrm{~K}$, a significant fraction of the oxygen is five-times ionised $\left(\mathrm{O}^{+5}\right.$ or O VI, IP $\left.=138 \mathrm{eV}\right)$. Six-times ionised oxygen $\left(\mathrm{O}^{+6}\right.$ or O VII, IP $=739 \mathrm{eV})$ and seven-times ionised oxygen $\left(\mathrm{O}^{+7}\right.$ or O VIII, IP $\left.=871 \mathrm{eV}\right)$ predominantly exist at higher temperatures in the range $3 \times 10^{5}-3 \times 10^{6} \mathrm{~K}$ and $3 \times 10^{6}-10^{7} \mathrm{~K}$, respectively. Figure 3 shows the ionisation fractions of the most important high ions of oxygen and neon, based on the CIE calculations of Sutherland and Dopita (1993); see also Kaastra et al. (2008-Chap. 9, this issue). High ions of other elements such as carbon, nitrogen, silicon and magnesium are less important for WHIM observations as their observable transitions trace lower temperature gas (e.g., C IV, Si IV) or the abundance of these elements in the intergalactic medium are too low. It is important to note at this point, that the discussed relation between ionisation state/fraction and gas temperature explicitly assumes that the gas is in an ionisation equilibrium. This may not be generally the case in the WHIM, however, 

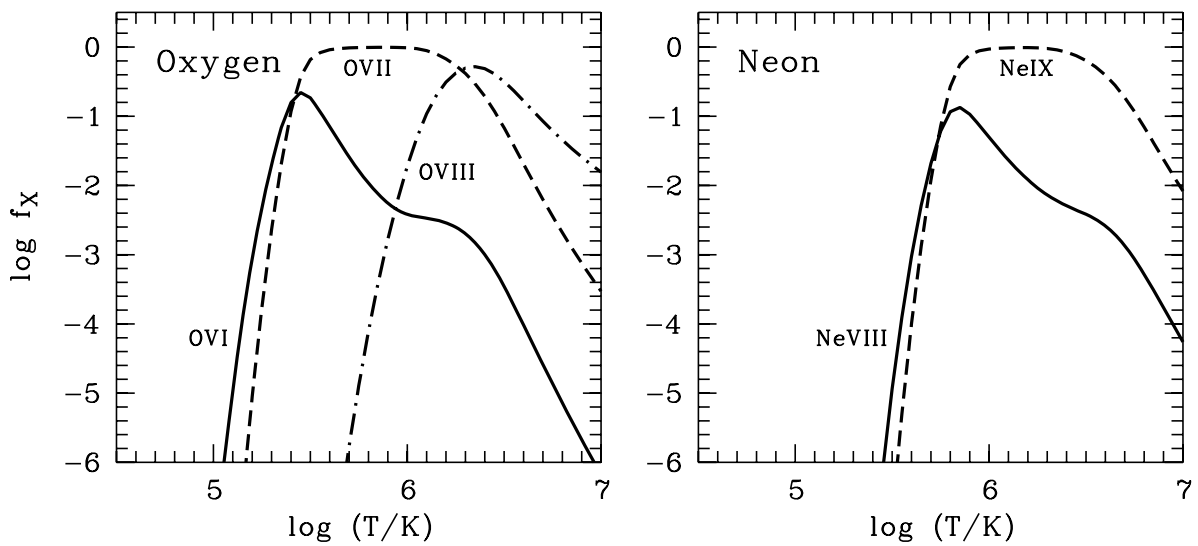

Fig. 3 CIE ion fractions of selected high ions of oxygen (O VI, O VII, O VIII; left panel) and neon (Ne VIII, Ne IX; right panel) in the WHIM temperature range $\log (T / \mathrm{K})=4.5-7.0$, based on calculations by Sutherland and Dopita (1993)

as the densities are generally very low. For instance, under particular non-equilibrium conditions the timescales for cooling, recombination, and ion/electron equilibration may differ significantly from each other (see for instance Bykov et al. 2008-Chap. 8, this issue). In such a case, the presence of high ions such as $\mathrm{O}$ VI and/or measured high-ion ratios would not serve as a reliable "thermometer" for the WHIM gas. In addition, WHIM filaments most likely neither are isothermal nor do they have a constant particle density. In fact, as WHIM simulations demonstrate, WHIM absorbers seem to represent a mix of cooler photoionised and hotter collisionally ionised gas with a substantial intrinsic density range. The absorption features from high ions arising in such a multi-phase medium therefore are generally difficult to interpret in terms of physical conditions and baryon budget.

In view of the high energies required to produce the high ions of oxygen and neon in combination with the spectral shape of the metagalactic background radiation (see Fig. 1), photoionisation of high metal ions in the WHIM is less important than for hydrogen. However, for O VI photoionisation is important at low redshifts in WHIM regions with very low densities or in systems located close to a strong local radiation source (e.g., in O VI systems associated with the background QSO). Note that at high redshift, most of the intervening $\mathrm{O}$ VI appears to be photoionised owing to the significantly higher intensity of the metagalactic background radiation in the early Universe (see Sect. 5.2).

\subsection{WHIM Absorption Signatures in the UV and X-Ray Band}

\subsubsection{UV Absorption}

As indicated in the previous subsection, five-times ionised oxygen $(\mathrm{O}$ VI) is by far the most important high ion to trace the WHIM at temperatures of $T \sim 3 \times 10^{5} \mathrm{~K}$ in the ultraviolet regime (assuming CIE, see above). Oxygen is a relatively abundant element and the two lithium-like $\left(1 s^{2} 2 s\right)^{2} S_{1 / 2} \rightarrow\left(1 s^{2} 2 p\right)^{2} P_{1 / 2,3 / 2}$ electronic transitions of O VI located in the FUV at 1031.9 and 1037.6 $\AA$ have large oscillator strengths $\left(f_{1031}=0.133, f_{1037}=0.066\right)$. Next to O VI, Ne VIII traces WHIM gas near $T \sim 7 \times 10^{5} \mathrm{~K}$ (in collisional ionisation equilibrium) and thus is possibly suited to complement the O VI measurements of the WHIM in a higher temperature regime. The two available Ne VIII lines are located in the extreme 
Table 1 Data on $\mathrm{O}$ and Ne high ions having observable absorption lines

\begin{tabular}{|c|c|c|c|c|c|}
\hline Ion & {$[\mathrm{X} / \mathrm{H}]^{\mathrm{a}}$} & $\begin{array}{l}\text { Ionisation } \\
\text { potential }[\mathrm{eV}]\end{array}$ & $\begin{array}{l}\text { Absorption } \\
\text { lines }[\AA ̊\end{array}$ & Band & $\begin{array}{l}\text { CIE temperature } \\
\text { range }\left[10^{6} \mathrm{~K}\right]\end{array}$ \\
\hline O VI & -3.34 & 138 & $\begin{array}{l}1031.926 \\
1037.617\end{array}$ & FUV & $0.2-0.5$ \\
\hline O VII & -3.34 & 739 & 21.602 & X-ray & $0.3-3.0$ \\
\hline O VIII & -3.34 & 871 & 18.969 & X-ray & $1.0-10.0$ \\
\hline Ne VIII & -4.16 & 239 & $\begin{array}{l}770.409 \\
780.324\end{array}$ & EUV & $0.5-1.3$ \\
\hline Ne IX & -4.16 & 1196 & 13.447 & X-ray & $0.6-6.3$ \\
\hline
\end{tabular}

${ }^{\mathrm{a}}[\mathrm{X} / \mathrm{H}]$ is the log of the number density of element $\mathrm{X}$ relative to hydrogen for Solar abundances, taken here from Asplund et al. (2004)

${ }^{\mathrm{b}}$ CIE models from Sutherland and Dopita (1993)

ultraviolet (EUV) at $770.4 \AA\left(f_{770}=0.103\right)$ and $780.3 \AA\left(f_{780}=0.051\right)$, allowing us to trace high-column density WHIM absorbers at redshifts $z>0.18$ with current FUV satellites such as FUSE. However, as the cosmic abundance of Ne VIII is relatively low, Ne VIII is not expected to be a particularly sensitive tracer of the WHIM at the S/N levels achievable with current UV spectrographs. The same argument holds for the high ion $\mathrm{Mg} \mathrm{X}$, which has two transitions in the EUV at even lower wavelengths $(\lambda \lambda 609.8,624.9 \AA)$. So far, only $\mathrm{O}$ VI and in one case Ne VIII has been observed in the WHIM at low redshift (see Sect. 3.2). Note that WHIM absorption features by O VI (and Ne VIII) are mostly unsaturated and the line profiles are fully or nearly resolved by current UV instruments such as FUSE and STIS, which provide spectral resolutions of $R=\lambda / \Delta \lambda \approx 20,000$ and 45,000, respectively. Table 1 summarises physical parameters of $\mathrm{O}$ and $\mathrm{Ne}$ high ions and their observable transitions in the UV and X-ray bands.

Four-times ionised nitrogen ( N v; I.P. is $98 \mathrm{eV}$ ) also is believed to trace predominantly collisionally ionised gas at temperatures near $T \sim 2 \times 10^{5} \mathrm{~K}$, but its lower cosmic abundance together with its deficiency in low metallicity environments due to nucleosynthesis effects (e.g., Pettini et al. 2002) makes it very difficult to detect in the WHIM. Other available strong high-ion transitions in the UV from carbon (C IV $\lambda \lambda$ 1548.2, $1550.8 \AA$ ) and silicon ( $\operatorname{Si}$ IV $\lambda \lambda$ $1393.8,1402.8 \AA$ ) are believed to trace mainly photoionised gas at temperatures $T<10^{5} \mathrm{~K}$, but not the shock-heated warm-hot gas at higher temperatures.

Next to high-ion absorption from heavy elements, recent UV observations (Richter et al. 2004; Sembach et al. 2004; Lehner et al. 2007) have indicated that WHIM filaments can be detected in Ly $\alpha$ absorption of neutral hydrogen. Although the vast majority of the hydrogen in the WHIM is ionised (by collisional processes and UV radiation), a tiny fraction ( $f_{\mathrm{HI}}<10^{-5}$, typically) of neutral hydrogen is expected to be present. Depending on the total gas column density of a WHIM absorber and its temperature, weak H I Ly $\alpha$ absorption at column densities $12.5 \leq \log N(\mathrm{H}$ I $) \leq 14.0$ may arise from WHIM filaments and could be used to trace the ionised hydrogen component. The Ly $\alpha$ absorption from WHIM filaments is very broad due to thermal line broadening, resulting in large Doppler parameters of $b>40$ $\mathrm{km} \mathrm{s}^{-1}$. Such lines are generally difficult to detect, as they are broad and shallow. High resolution, high S/N FUV spectra of QSOs with smooth background continua are required to successfully search for broad Ly $\alpha$ absorption in the low-redshift WHIM. STIS installed 
on the HST is the only instrument that has provided such data, but due to the instrumental limitations of space-based observatories, the number of QSO spectra adequate for searching for WHIM broad Ly $\alpha$ absorption (in the following abbreviated as "BLA") is very limited.

The $b$ values of the BLAs are assumed to be composed of a thermal component, $b_{\mathrm{th}}$, and a non-thermal component, $b_{\mathrm{nt}}$, in the way that

$$
b=\sqrt{b_{\mathrm{th}}^{2}+b_{\mathrm{nt}}^{2}} .
$$

The non-thermal component may include processes like macroscopic turbulence, unresolved velocity-components, and others (see Richter et al. 2006a for a detailed discussion). The contribution of the thermal component to $b$ depends on the gas temperature:

$$
b_{\mathrm{th}}=\sqrt{\frac{2 k T}{m}} \approx 0.13 \sqrt{\frac{T}{A}} \mathrm{~km} \mathrm{~s}^{-1},
$$

where $T$ is in $\mathrm{K}, k$ is the Boltzmann constant, $m$ is the particle mass, and $A$ is the atomic weight. For the shock-heated WHIM gas with $\log T \geq 5$ one thus expects $b_{\text {th }} \geq 40 \mathrm{~km} \mathrm{~s}^{-1}$. The non-thermal broadening mechanisms are expected to contribute to some degree to the total $b$ values in WHIM absorbers (see Richter et al. 2006a), so that the measured $b$ value of a BLA provides only an upper limit for the temperature of the gas.

\subsubsection{X-Ray Absorption}

The highest ionisation phase of the WHIM will produce and absorb line radiation primarily in the He- and $\mathrm{H}$-like ions of the low- $\mathrm{Z}$ elements $(\mathrm{C}, \mathrm{N}, \mathrm{O}, \mathrm{Ne})$, and possibly in the L-shell ions of Fe. In practice, much of the attention is focused on oxygen, because of its relatively high abundance, and because the strongest resonance lines in $\mathrm{He}$ - and $\mathrm{H}$-like $\mathrm{O}$ are in a relatively 'clean' wavelength band. For reference, the Ly $\alpha$ transitions of C VI, N VII, O VIII, and $\mathrm{Ne} X$ occur at $33.7360,24.7810,18.9689$, and $12.1339 \AA$, respectively (wavelengths of the $1 s-2 p_{1 / 2,3 / 2}$ doublet weighted with oscillator strength; Johnson and Soff 1985). The Helike ions $\mathrm{C} \mathrm{V}, \mathrm{N}$ VI, O VII, and Ne IX have their strongest transition, the $n=1-2$ resonance line, at 40.2674, 28.7800, 21.6015, and 13.4473 $\AA$ (Drake 1988; see also Table 1). Data on the higher order series members can be found in Verner et al. (1996). As far as the Fe L shell ions are concerned, the most likely transition to show up would be the strongest line in Ne-like Fe XVII, $n=2 p-3 d \lambda 15.014 \AA$. In addition, all lower ionisation stages of $\mathrm{C}, \mathrm{N}$, $\mathrm{O}$, and $\mathrm{Ne}$ (with the exception of neutral $\mathrm{Ne}$ of course) can also absorb by $n=1-2$; the strongest of these transitions would be $1 s-2 p$ in O VI at $22.019 \AA$ (Schmidt et al. 2004). Likewise, the lower ionisation stages of Fe could in principle produce $n=2-3$ absorption.

The thermal widths of all these transitions will be very small, requiring resolving powers of order $R \sim 10000(\mathrm{C}, \mathrm{N}, \mathrm{O}, \mathrm{Ne})$ for gas temperatures of order $10^{6} \mathrm{~K}$ to be resolved; for $\mathrm{Fe}$, the requirement is even higher, by a factor $\sim 2$. As we will see, for practical reasons, these requirements exceed the current capabilities of astrophysical X-ray spectroscopy by a large factor. Due to the small Doppler broadening (ignoring turbulent velocity fields for now), the lines will rapidly saturate. For He- and $\mathrm{H}$-like $\mathrm{O}$ resonance line absorption, saturation sets in at an equivalent width of order $1 \mathrm{~m} \AA$ (Kaastra et al. 2008-Chap. 9, this issue) or column densities of order a few times $10^{14}$ ions $\mathrm{cm}^{-2}$. The challenge, therefore, for X-ray spectroscopy presented by the IGM is to detect small equivalent width, near-saturation lines that are unresolved. 


\subsection{The Baryon Content of the WHIM as Measured by UV and X-Ray Absorbers}

One important result from absorption line measurements of the WHIM in the UV is the observed number density of WHIM absorbers, usually expressed as $\mathrm{d} N / \mathrm{d} z$, the number of absorbers per unit redshift. For instance, from recent measurements with FUSE and HST/STIS one finds for $\mathrm{O}$ VI absorbers and Broad Ly $\alpha$ Absorbers at $z \approx 0$ values of $\mathrm{d} N / \mathrm{d} z(\mathrm{O}$ VI) $\approx 20$ and $\mathrm{d} N / \mathrm{d} z(\mathrm{BLA}) \approx 30$ (see Sect. 3.2). Currently, the WHIM absorber density is only measurable in the UV, since in the X-ray band both the observed number of WHIM absorption lines and the available redshift path for WHIM observations is too small to derive statistically significant values of $\mathrm{d} N / \mathrm{d} z(\mathrm{O}$ VII $)$ and $\mathrm{d} N / \mathrm{d} z(\mathrm{O}$ VIII).

A particularly interesting question now is, how the observed number density of high-ion lines or BLAs translates into an estimate of the cosmological baryon mass density of the WHIM, $\Omega_{\mathrm{b}}$ (WHIM). To obtain such an estimate of the baryon content of the WHIM from UV and X-ray absorption measurements one has to consider two main steps. First, one needs to transform the observed column densities of the high ions (e.g., O vI, O VII, O VIII) into a total gas column density by modelling the ionisation conditions in the gas. In a second step, one then integrates over the total gas column densities of all observed WHIM absorbers along the given redshift path and from that derives $\Omega_{\mathrm{b}}$ (WHIM) for a chosen cosmology. Throughout the paper we will assume a $\Lambda$ CDM cosmology with $H_{0}=70 \mathrm{~km} \mathrm{~s}^{-1} \mathrm{Mpc}^{-1}$, $\Omega_{\Lambda}=0.7, \Omega_{\mathrm{m}}=0.3$, and $\Omega_{\mathrm{b}}=0.045$. For the first step the uncertainty lies in the estimate of the ionisation fraction of hydrogen of the WHIM. For this, it is usually assumed that the WHIM is in collisional ionisation equilibrium, but photoionisation and non-equilibrium conditions may play a significant role. In the case of using metal ions such as O vI the unknown oxygen abundance $(\mathrm{O} / \mathrm{H})$ of the gas introduces an additional uncertainty (see below) for the estimate of $\Omega_{\mathrm{b}}$ (WHIM). For the second step, it is important to have a large enough sample of WHIM absorption lines and a sufficient total redshift path along different directions in order to handle statistical errors and the problem of cosmic variance. As mentioned earlier, these requirements currently are fulfilled only for the UV absorbers.

The cosmological mass density $\Omega_{\mathrm{b}}$ of O VI absorbers (and, similarly, for other high ions) in terms of the current critical density $\rho_{\mathrm{c}}$ can be estimated by

$$
\Omega_{\mathrm{b}}(\mathrm{O} \mathrm{vI})=\frac{\mu m_{\mathrm{H}} H_{0}}{\rho_{\mathrm{c}} c} \sum_{i j} \frac{N(\mathrm{O} \mathrm{vI})_{i j}}{f_{\mathrm{O} \mathrm{VI}, i j}(\mathrm{O} / \mathrm{H})_{i j} \Delta X_{j}} .
$$

In this equation, $\mu=1.3$ is the mean molecular weight, $m_{\mathrm{H}}=1.673 \times 10^{-27} \mathrm{~kg}$ is the mass per hydrogen atom, $H_{0}$ is the adopted local Hubble constant, and $\rho_{\mathrm{c}}=3 H_{0}{ }^{2} / 8 \pi G$ is the current critical density. The index $i$ denotes an individual high-ion absorption system along a line of sight $j$. Each measured high-ion absorption system $i$ is characterised by its measured ion column density (e.g., $N(\mathrm{O} \text { VI })_{i j}$ ), the ionisation fraction of the measured ion (e.g., $f_{\mathrm{OVI}, i j}$ ), and the local abundance of the element measured compared to hydrogen (e.g., the local oxygen-to-hydrogen ratio, by number). Each line of sight $j$ has a characteristic redshift range $\Delta z$ in which high-ion absorption may be detected. The corresponding comoving path length $\Delta X$ available for the detection of WHIM high-ion absorbers then is given by:

$$
\Delta X_{j}=(1+z)^{2}\left[\Omega_{\Lambda}+\Omega_{\mathrm{m}}(1+z)^{3}\right]^{-0.5} \Delta z_{j} .
$$

In analogy, we can write for the cosmological mass density of the BLAs:

$$
\Omega_{\mathrm{b}}(\mathrm{BLA})=\frac{\mu m_{\mathrm{H}} H_{0}}{\rho_{\mathrm{c}} c} \sum_{i j} \frac{N(\mathrm{HI})_{i j}}{f_{\mathrm{H}, i j} \Delta X_{j}} .
$$


As can be easily seen, the advantage of using BLAs for deriving the WHIM mass density is that the metallicity of the gas is unimportant for the determination of $\Omega_{\mathrm{b}}$. The disadvantage is, however, that the ionisation corrections are very large and uncertain, since they are determined indirectly from the BLA line widths (see Sect. 2.2.1).

\section{UV Measurements of the WHIM}

\subsection{Past and Present UV Instruments}

The first and second generations of space based UV spectrographs such as Copernicus and the International Ultraviolet Explorer (IUE) did not have sufficient sensitivity to systematically study intervening absorption in the intergalactic medium along a large number of sightlines. The early low- and intermediate resolution spectrographs installed on the Hubble Space Telescope (HST), namely the Faint Object Spectrograph (FOS) and the Goddard High Resolution Spectrograph (GHRS), were used to study the properties of the local Ly $\alpha$ forest and intervening metal-line systems (e.g., Stocke et al. 1995; Shull et al. 1998). While intervening O VI absorption has been detected with these instruments (e.g., Tripp et al. 1998), the concept of a warm-hot intergalactic gas phase was not really established at that time. With the implementation of the high-resolution capabilities of the Space Telescope Imaging Spectrograph (STIS) installed on HST the first systematic analyses of WHIM O VI absorbers as significant low-redshift baryon reservoirs came out in 2000 (see Tripp et al. 2000), thus relatively soon after the importance of a shock-heated intergalactic gas phase was realised in cosmological simulations for the first time (e.g., Cen and Ostriker 1999; Davé et al. 2001). The STIS echelle spectrograph together with the E140M grating provides a high spectralresolution of $R \approx 45000$, corresponding to a velocity resolution of $\sim 7 \mathrm{~km} \mathrm{~s}^{-1}$ in the STIS E140M wavelength band between 1150 and $1730 \AA$ (e.g., Kimble et al. 1998; Woodgate 1998). An example for a STIS quasar spectrum with intervening hydrogen and metal-line absorption is shown in Fig. 4. Note that at the spectral resolution of the STIS E140M grating all intergalactic absorption lines (i.e., hydrogen and metal lines) are fully resolved. In 1999, the Far Ultraviolet Spectroscopic Explorer (FUSE) became available, covering the wavelength range between 912 and $1187 \AA$. Equipped with a Rowland-type spectrograph providing a medium spectral resolution of $R \approx 20000$ (FWHM $\sim 20 \mathrm{~km} \mathrm{~s}^{-1}$ ) FUSE is able to observe extragalactic UV background sources brighter than $V=16.5$ mag with acceptable integration time and signal-to-noise (S/N) ratios (for a description of FUSE see Moos et al. 2000; Sahnow et al. 2000). With this resolution, FUSE is able to resolve the broader intergalactic absorption from the H I Lyman series, while most of the narrow metalline absorbers remain just unresolved. This is not a problem for O VI WHIM studies with FUSE, however, since the spectral resolution is very close to the actual line widths and the O VI absorption usually is not saturated. FUSE complements the STIS instruments at lower wavelengths down to the Lyman limit and consequently combined FUSE and STIS spectra of $\sim 15$ low redshift QSOs and AGN have been used to study the low-redshift WHIM via intervening O VI and BLA absorption (see Tripp et al. 2007 and references therein). Unfortunately, since 2006/2007 both STIS and FUSE are out of commission due to technical problems.

Fresh spectroscopic UV data from WHIM absorption line studies will become available once the Cosmic Origins Spectrograph (COS) will be installed on HST during the next HST 


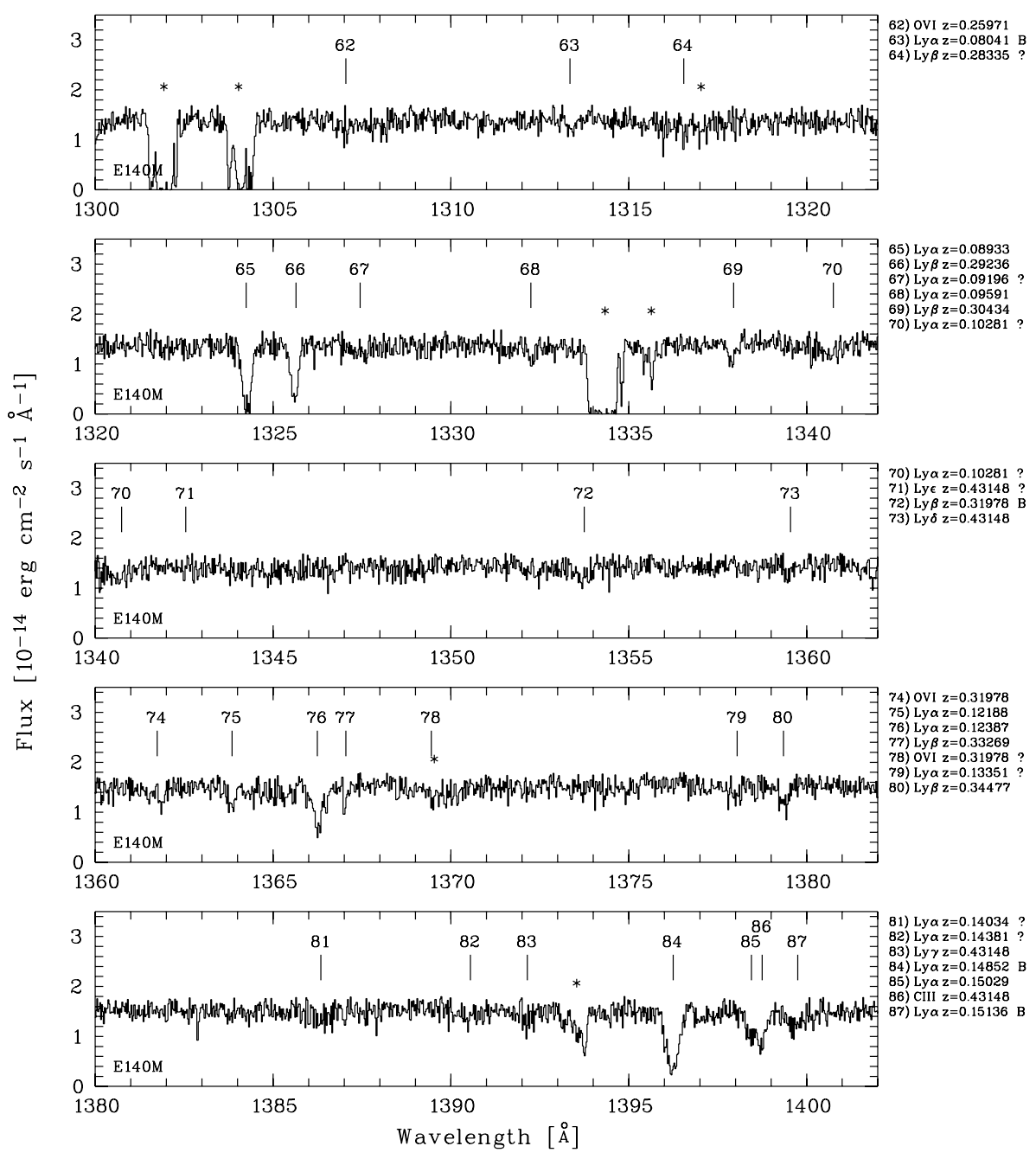

Fig. 4 STIS spectrum of the quasar PG 1259+593 in the wavelength range between 1300 and $1400 \AA$. Next to absorption from the local Ly $\alpha$ forest and gas in the Milky Way there are several absorption features that most likely are related to highly ionised gas in the WHIM. Absorption from five-times ionised oxygen (O VI) is observed at $z=0.25971$ and $z=0.31978$. Broad H I Ly $\alpha$ and Ly $\beta$ absorption is detected at $z=0.08041,0.09196,0.10281,0.13351,0.14034,0.14381,0.14852,0.15136$, and $z=0.31978$. From Richter et al. (2004)

service mission (SM-4), which currently is scheduled for late 2008. COS will observe in the UV wavelength band between 1150 and $3000 \AA$ at medium resolution $(R \approx 20000)$. COS has been designed with maximum effective area as the primary constraint: it provides more than an order of magnitude gain in sensitivity over previous HST instruments. Due to its very high sensitivity, COS thus will be able to observe hundreds of low- and intermediate redshift QSOs and AGN and thus will deliver an enormous data archive to study the properties of WHIM UV absorption lines systems in great detail (see also Paerels et al. 2008-Chap. 19, this issue). 

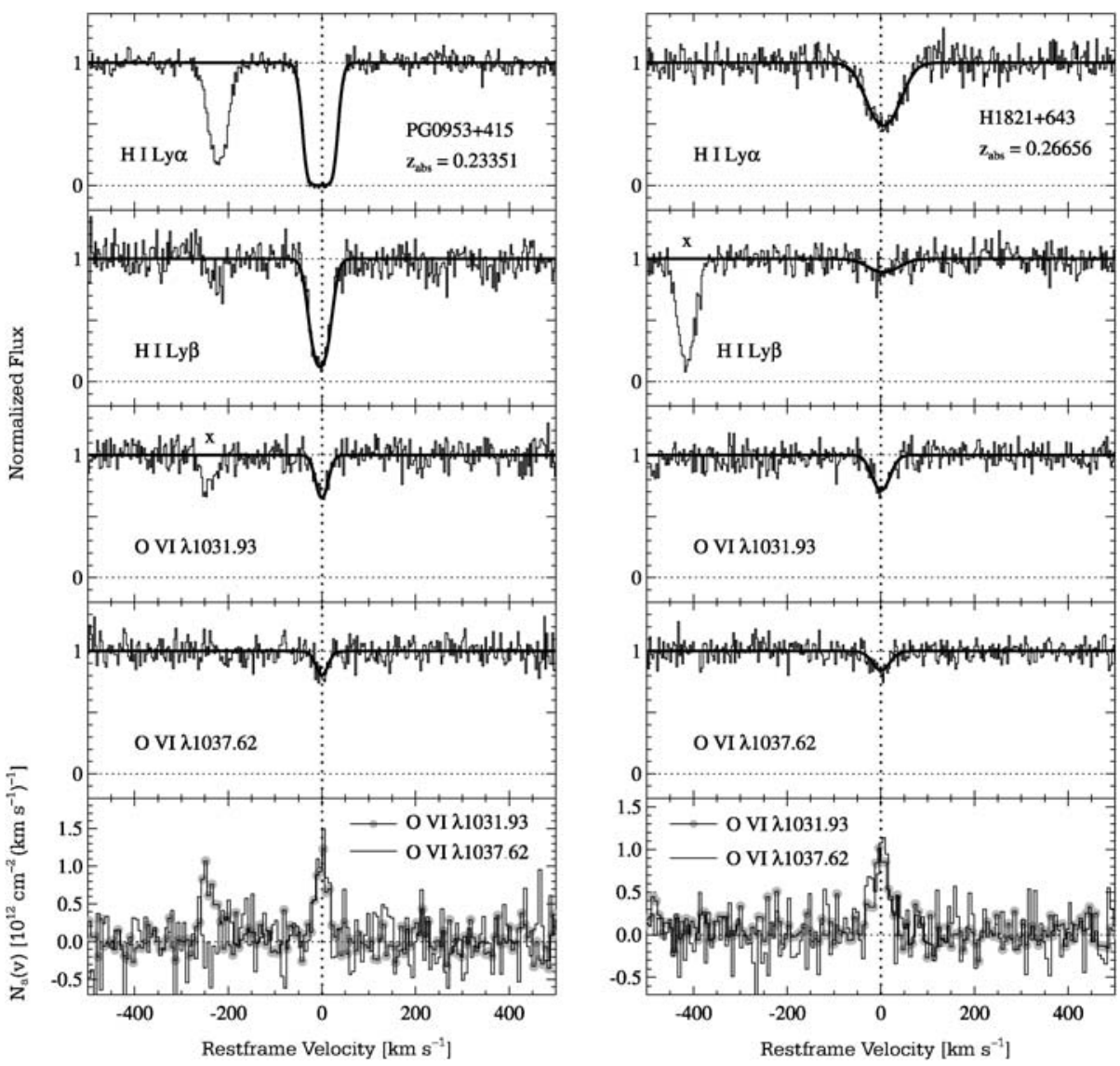

Fig. 5 Examples for $\mathrm{HI}$ and O VI absorption in two absorption systems at $z=0.23351$ and $z=0.6656$ towards PG $0953+415$ and $\mathrm{H} 1821+643$, respectively, plotted on a rest frame velocity scale (observed with STIS). Adapted from Tripp et al. (2007)

\subsection{Intervening WHIM Absorbers at Low Redshift}

We start with the $\mathrm{O}$ VI absorbers which are believed to trace the low-temperature tail of the WHIM at $T<5 \times 10^{5} \mathrm{~K}$. Up to now, more than 50 detections of intervening $\mathrm{O}$ VI absorbers at $z<0.5$ have been reported in the literature (e.g., Tripp et al. 2000; Oegerle et al. 2000; Chen and Prochaska 2000; Savage et al. 2002; Richter et al. 2004; Sembach et al. 2004; Savage et al. 2005; Danforth and Shull 2005; Tripp et al. 2007). All of these detections are based on FUSE and STIS data. Figure 5 shows two examples for intervening O VI absorption at $z=0.23351$ and $z=0.26656$ in the direction of PG $0953+415$ and $\mathrm{H} 1821+643$, as observed with STIS. The most recent compilation of low-redshift intervening O VI absorbers is that of Tripp et al. (2007), who have analysed 16 sightlines toward low-redshift QSOs observed with STIS and FUSE along a total redshift path of $\Delta z \approx 3$. They find a total of 53 intervening O VI absorbers (i.e., they are not within $5000 \mathrm{~km} \mathrm{~s}^{-1}$ of $z_{\mathrm{QSO}}$ ) comprised of 78 individual absorption components. ${ }^{1}$ The measurements imply a number density of O VI ab-

\footnotetext{
${ }^{1}$ I.e., some of the O VI systems have velocity sub-structure.
} 
sorbing systems per unit redshift of $\mathrm{d} N_{\mathrm{O} \text { VI }} / \mathrm{d} z \approx 18 \pm 3$ for equivalent widths $W_{\lambda} \geq 30 \mathrm{~m} \AA$. The corresponding number density of $\mathrm{O}$ VI absorption components is $\mathrm{d} N_{\mathrm{O} \text { VI }} / \mathrm{d} z \approx 25 \pm 3$. These values are slightly higher than what is found by earlier analyses of smaller O VI samples (Danforth and Shull 2005), but lie within the cited $2 \sigma$ error ranges. The discrepancy between the measured O VI number densities probably is due to the different approaches of estimating the redshift path $\Delta z$ along which the $\mathrm{O}$ VI absorption takes place. If one assumes that the gas is in a collisional ionisation equilibrium, i.e., that $\sim 20$ percent of the oxygen is present in the form of $\mathrm{O}$ VI $\left(f_{\mathrm{OVI}} \leq 0.2\right)$, and further assumes that the mean oxygen abundance is 0.1 Solar, the measured number density of $\mathrm{O} V \mathrm{VI}$ absorbers corresponds to a cosmological mass density of $\Omega_{\mathrm{b}}(\mathrm{O} \mathrm{VI}) \approx 0.0020-0.0030 h_{70}{ }^{-1}$. These values imply that intervening $\mathrm{O}$ VI absorbers trace $\sim 5-7$ percent of the total baryon mass in the local Universe. For the interpretation of $\Omega_{\mathrm{b}}(\mathrm{O} \mathrm{VI})$ it has to be noted that $\mathrm{O}$ VI absorption traces collisionally ionised gas at temperatures around $3 \times 10^{5} \mathrm{~K}$ (and also low-density, photoionised gas at lower temperatures), but not the million-degree gas phase which probably contains the majority of the baryons in the WHIM.

The recent analysis of Tripp et al. (2007) indicates, however, that this rather simple conversion from measured $\mathrm{O}$ VI column densities to $\Omega_{\mathrm{b}}(\mathrm{O} \mathrm{VI})$ may not be justified in general, as the CIE assumption possibly breaks down for a considerable fraction of the $\mathrm{O}$ VI systems. From the measured line widths of the $\mathrm{HI}$ Ly $\alpha$ absorption that is associated with the $\mathrm{O}$ VI Tripp et al. conclude that $\sim 40$ percent of their O VI systems belong to cooler, photoionised gas with $T<10^{5} \mathrm{~K}$, possibly not at all associated with shock-heated warm-hot gas. In addition, about half of the intervening O VI absorbers arise in rather complex, multi-phase systems that can accommodate hot gas at relatively low metallicity. It thus appears thatwithout having additional information about the physical conditions in each O VI absorberthe estimate of the baryon budget in intervening O VI systems is afflicted with rather large systematic uncertainties.

In high-column density O VI systems at redshifts $z>0.18$, such desired additional information may be provided by the presence or absence of Ne VIII (see Sect. 2.2.1), which in CIE traces gas at $T \sim 7 \times 10^{5} \mathrm{~K}$. Toward the quasar PG 1259+593 Richter et al. (2004) have reported a tentative detection of Ne VIII absorption at $\sim 2 \sigma$ significance in an $\mathrm{O}$ VI absorber at $z \approx 0.25$. The first secure detection of intervening Ne VIII absorption (at $\sim 4 \sigma$ significance) was presented by Savage et al. (2005) in a multi-phase O VI absorption system at $z \approx 0.21$ in the direction of the quasar HE 0226-4110. The latter authors show that in this particular absorber the high-ion ratio Ne VIII/O VI $=0.33$ is in agreement with gas in CIE at temperature of $T \sim 5 \times 10^{5} \mathrm{~K}$. With future high $\mathrm{S} / \mathrm{N}$ absorption line data of lowredshift QSOs (as will be provided by COS) it is expected that the number of detections of WHIM Ne VIII absorbers will increase substantially, so that an important new diagnostic will become available for the analysis of high-ion absorbers.

One other key aspect in understanding the distribution and nature of intervening O VI systems concerns their relation to the large-scale distribution of galaxies. Combining FUSE data of $37 \mathrm{O}$ VI absorbers with a database of more than a million galaxy positions and redshifts, Stocke et al. (2006) find that all of these O VI systems lie within $800 h_{70}{ }^{-1} \mathrm{kpc}$ of the nearest galaxy. These results suggest that $\mathrm{O}$ VI systems preferentially arise in the immediate circumgalactic environment and extended halos of galaxies, where the metallicity of the gas is expected to be relatively high compared to regions far away from galactic structures. Some very local analogs of intervening O VI systems thus may be the O VI high-velocity clouds in the Local Group that are discussed in the next subsection. Due to apparent strong connection between intervening O VI systems and galactic structures and a resulting galaxy/metallicity bias problem it is of great interest to consider other tracers of warm-hot gas, which are 
independent of the metallicity of the gas. The broad hydrogen Ly $\alpha$ absorbers - as will be discussed in the following - therefore represent an important alternative for studying the WHIM at low redshift.

As described in Sect. 2.2.1, BLAs represent H I Ly $\alpha$ absorbers with large Doppler parameters $b>40 \mathrm{~km} \mathrm{~s}^{-1}$. If thermal line broadening dominates the width of the absorption, these systems trace the WHIM at temperatures between $10^{5}$ and $10^{6} \mathrm{~K}$, typically (note that for most systems with $T>10^{6} \mathrm{~K}$ BLAs are both too broad and too shallow to be unambiguously identified with the limitations of current UV spectrographs). The existence of $\mathrm{HI} \mathrm{Ly} \alpha$ absorbers with relatively large line widths has been occasionally reported in earlier absorption-line studies of the local intergalactic medium (e.g., Tripp et al. 2001; Bowen et al. 2002). Motivated by the rather frequent occurrence of broad absorbers along QSO sightlines with relatively large redshift paths, the first systematic analyses of BLAs in STIS low- $z$ data were carried out by Richter et al. (2004) and Sembach et al. (2004). Richter et al. (2006a) have inspected four sightlines observed with STIS towards the quasars PG $1259+593\left(z_{\mathrm{em}}=0.478\right), \mathrm{PG} 1116+215\left(z_{\mathrm{em}}=0.176\right), \mathrm{H} 1821+643$ $\left(z_{\mathrm{em}}=0.297\right)$, and PG 0953+415 $\left(z_{\mathrm{em}}=0.239\right)$ for the presence of BLAs and they identified a number of good candidates. Their study implies a BLA number density per unit redshift of $\mathrm{d} N_{\mathrm{BLA}} / \mathrm{d} z \approx 22-53$ for Doppler parameters $b \geq 40 \mathrm{~km} \mathrm{~s}^{-1}$ and above a sensitivity limit of $\log \left(N\left(\mathrm{~cm}^{-2}\right) / b\left(\mathrm{~km} \mathrm{~s}^{-1}\right)\right) \geq 11.3$. The large range for $\mathrm{d} N_{\mathrm{BLA}} / \mathrm{d} z$ partly is due to the uncertainty about defining reliable selection criteria for separating spurious cases from good broad Ly $\alpha$ candidates (see discussions in Richter et al. 2004, 2006a and Sembach et al. 2004). Transforming the number density $\mathrm{d} N_{\mathrm{BLA}} / \mathrm{d} z$ into a cosmological baryonic mass density, Richter et al. (2006a) obtains $\Omega_{\mathrm{b}}$ (BLA) $\geq 0.0027 h_{70}{ }^{-1}$. This limit is about 6 percent of the total baryonic mass density in the Universe expected from the current cosmological models (see above), and is comparable with the value derived for the intervening O VI absorbers (see above). Examples for several BLAs in the STIS spectrum of the quasar H 1821+643 are shown in Fig. 6.

More recently, Lehner et al. (2007) have analysed BLAs in low-redshift STIS spectra along seven sightlines. They find a BLA number density of $\mathrm{d} N_{\mathrm{BLA}} / \mathrm{d} z=30 \pm 4$ for $b=40-150 \mathrm{~km} \mathrm{~s}^{-1}$ and $\log N(\mathrm{HI})>13.2$ for the redshift range $z=0-0.4$. They conclude that BLAs host at least 20 percent of the baryons in the local Universe, while the photoionised Ly $\alpha$ forest, which produces a large number of narrow Ly $\alpha$ absorbers (NLAs), contributes with $\sim 30$ percent to the total baryon budget. In addition, Prause et al. (2007) have investigated the properties of BLAs at intermediate redshifts $(z=0.9-1.9)$ along five other quasars using STIS high- and intermediate-resolution data. They find a number density of reliably detected BLA candidates of $\mathrm{d} N_{\mathrm{BLA}} / \mathrm{d} z \approx 14$ and obtain a lower limit of the contribution of BLAs to the total baryon budget of $\sim 2$ percent in this redshift range. The frequency and baryon content of BLAs at intermediate redshifts obviously is lower than at $z=0$, indicating that at intermediate redshifts shock-heating of the intergalactic gas from the infall in large-scale filaments is not yet very efficient. This is in line with the predictions from cosmological simulations.

\subsection{The Milky Way Halo and Local Group Gas}

One primary goal of the FUSE mission was to constrain the distribution and kinematics of hot gas in the thick disk and lower halo of the Milky Way by studying the properties of Galactic O VI absorption systems at radial velocities $\left|v_{\mathrm{LSR}}\right| \leq 100 \mathrm{~km} \mathrm{~s}^{-1}$ (Savage et al. 2000, 2003; Wakker et al. 2003). However, as the FUSE data unveil, O VI absorption associated with Milky Way gas is observed not only at low velocities but also at $\left|v_{\mathrm{LSR}}\right|>100$ 


\section{H $1821+643$}

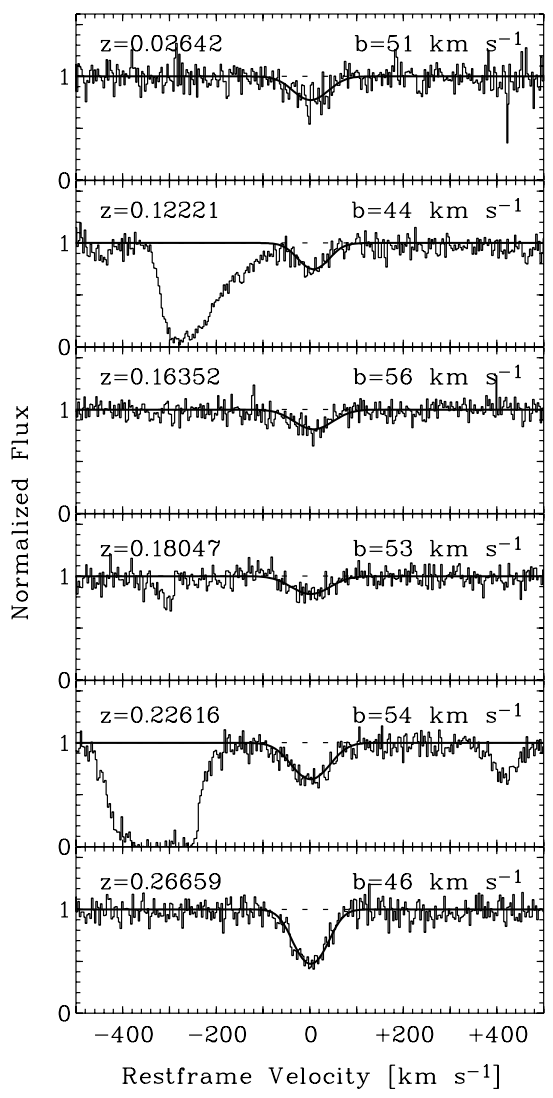

PG $\quad 0953+415$

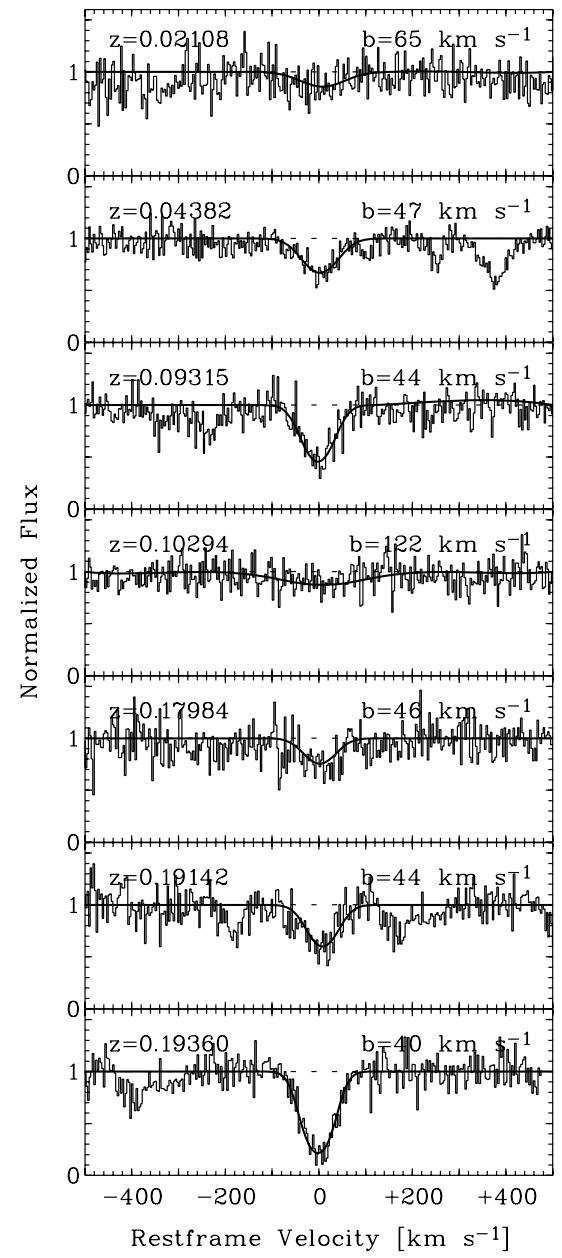

Fig. 6 Broad Lyman $\alpha$ absorbers towards the quasars H 1821+643 and PG 0953+415 (STIS observations), plotted on a rest frame velocity scale. If thermal line broadening dominates the width of the absorption, these systems trace the WHIM at temperatures between $10^{5}$ and $10^{6} \mathrm{~K}$. From Richter et al. (2006a)

$\mathrm{km} \mathrm{s}^{-1}$ (Sembach et al. 2003). The topic of cool and hot gas in the halo of the Milky Way recently has been reviewed by Richter (2006c). These detections imply that next to the Milky Way's hot "atmosphere" (i.e., the Galactic Corona) individual pockets of hot gas exist that move with high velocities through the circumgalactic environment of the Milky Way. Such high-velocity O VI absorbers may contain a substantial fraction of the baryonic matter in the Local Group in the form of warm-hot gas and thus-as discussed in the previous subsection-possibly represent the local counterparts of some of the intervening O VI absorbers observed towards low-redshift QSOs.

From their FUSE survey of high-velocity O VI absorption Sembach et al. (2003) find that probably more than 60 percent of the sky at high velocities is covered by ionised hydrogen (associated with the O VI absorbing gas) above a column density level of $\log N(\mathrm{H} \mathrm{II})=18$, 
assuming a metallicity of the gas of 0.2 Solar. Some of the high-velocity O VI detected with FUSE appears to be associated with known high-velocity H I $21 \mathrm{~cm}$ structures (e.g., the high-velocity clouds complex A, complex C, the Magellanic Stream, and the Outer Arm). Other high-velocity O VI features, however, have no counterparts in $\mathrm{H}$ I $21 \mathrm{~cm}$ emission. The high radial velocities for most of these $\mathrm{O}$ VI absorbers are incompatible with those expected for the hot coronal gas (even if the coronal gas motion is decoupled from the underlying rotating disk). A transformation from the Local Standard of Rest to the Galactic Standard of Rest and the Local Group Standard of Rest velocity reference frames reduces the dispersion around the mean of the high-velocity O VI centroids (Sembach et al. 2003; Nicastro et al. 2003). This can be interpreted as evidence that some of the O VI high-velocity absorbers are intergalactic clouds in the Local Group rather than clouds directly associated with the Milky Way. However, it is extremely difficult to discriminate between a Local Group explanation and a distant Galactic explanation for these absorbers. The presence of intergalactic O VI absorbing gas in the Local Group is in line with theoretical models that predict that there should be a large reservoir of hot gas left over from the formation of the Local Group (see, e.g., Cen and Ostriker 1999).

It is unlikely that the high-velocity O VI is produced by photoionisation. Probably, the gas is collisionally ionised at temperatures of several $10^{5} \mathrm{~K}$. The $\mathrm{O}$ VI then may be produced in the turbulent interface regions between very hot $\left(T>10^{6} \mathrm{~K}\right)$ gas in an extended Galactic Corona and the cooler gas clouds that are moving through this hot medium (see Sembach et al. 2003). Evidence for the existence of such interfaces also comes from the comparison of absorption lines from neutral and weakly ionised species with absorption from high ions like O vi (Fox et al. 2004).

\section{X-Ray Measurements of the WHIM}

\subsection{Past and Present X-Ray Instruments}

With the advent of the Chandra and XMM-Newton observatories, high resolution X-ray spectroscopy of a wide variety of cosmic sources became feasible for the first time. Among the possible results most eagerly speculated upon was the detection of intergalactic absorption lines from highly ionised metals in the continuum spectra of bright extragalactic sources. After all, one of the most striking results from the Einstein observatory had been the detection of a very significant broad absorption feature at $\sim 600 \mathrm{eV}$ in the spectrum of PKS 2155-304 with the Objective Grating Spectrometer (Canizares and Kruper 1984). Ironically, if interpreted as intergalactic $\mathrm{H}$-like $\mathrm{O}$ Ly $\alpha$ absorption, redshifted and broadened by the expansion of the Universe, the strength of the feature implied the presence of a highly ionised IGM of near-critical density, a possibility that has of course definitively been discounted since then.

The High Energy Transmission Grating Spectrometer (HETGS; Canizares et al. 2005) and the Low Energy Transmission Grating Spectrometer (LETGS; Brinkman et al. 2000) on Chandra, and the Reflection Grating Spectrometer (RGS) on XMM-Newton (den Herder et al. 2001) were the first instruments to provide sensitivity to weak interstellar and intergalactic X-ray absorption lines. The 'traditional' ionisation detectors (proportional counters, CCD's) do not have sufficient energy resolution for this application. But the angular resolution provided by an X-ray telescope can be used to produce a high resolution spectrum, by the use of diffracting elements. Laboratory X-ray spectroscopy is typically performed with crystal diffraction spectrometers, and the use of crystal spectrometers for general use 
in astrophysics was pioneered on the Einstein observatory (e.g., Canizares et al. 1979). The Focal Plane Crystal Spectrometer indeed detected the first ever narrow X-ray absorption line in a cosmic source, the $1 s-2 p$ absorption by neutral oxygen in the interstellar medium towards the Crab (Canizares and Kruper 1984). Previous grating spectrometers (the Objective Grating Spectrometer on Einstein and the two Transmission Grating Spectrometers on EXOSAT) had only limited resolution and sensitivity. But there is no fundamental limit to the resolution of a diffraction grating spectrometer, and the high angular resolution of the Chandra telescope has allowed for high resolution spectroscopy using transmission gratings. The focusing optics on XMM-Newton have more modest angular resolution, but they are used with a fixed array of grazing incidence reflection gratings, which produce very large dispersion angles and thus high spectral resolution. The HETGS provides a spectral resolution of $\Delta \lambda=0.0125 \AA$ over the $\approx 1.5-15 \AA$ band with the high line density grating, and $\Delta \lambda=0.025 \AA$ over the $2-20 \AA$ band with the medium line density grating. The LETGS has $\Delta \lambda=0.05 \AA$ over the $\approx 2-170 \AA$ band, while the RGS has $\Delta \lambda=0.06 \AA$ over the $5-38 \AA$ band. These numbers translate to resolving powers of $R=400-1500$ in the $\mathrm{O} \mathrm{K}$ band, and with a sufficiently bright continuum source, one should be able to detect equivalent widths of order $0.1-0.5 \mathrm{eV}(5-20 \mathrm{~m} \AA$ ), or below in spectra with very high signal-to-noise. The predictions for $\mathrm{H}$ - and $\mathrm{He}$-like $\mathrm{O}$ resonance absorption line strengths are generally smaller than these thresholds, but not grossly so, and so a search for intergalactic $\mathrm{O}$ was initiated early on. Given that the current spectrometers are not expected to resolve the absorption lines, the only freedom we have to increase the sensitivity of the search is to increase the signal-to-noise ratio in the continuum, and it becomes crucial to find suitable, very bright sources, at redshifts that are large enough that there is a reasonable a priori probability of finding a filament with detectable line absorption.

As we will see when we discuss the results of the observational searches for X-ray absorption lines, the problem is made considerably more difficult by the very sparseness of the expected absorption signature. Frequently, when absorption features of marginal statistical significance are detected in astrophysical data, plausibility is greatly enhanced by simple, unique spectroscopic arguments. For instance, for all plausible parameter configurations, an absorbing gas cloud detected in $\mathrm{N} v$ should also produce detectable $\mathrm{C}$ IV absorption; or, both members of a doublet should appear in the correct strength ratio if unsaturated. In the early stages of Ly $\alpha$ forest astrophysics, it was arguments of this type, rather than the crossing of formal statistical detection thresholds alone, that guided the field (e.g., Lynds 1971). But for the 'X-ray Forest' absorption, we expect a very different situation. The detailed simulations confirm what simple analytical arguments had suggested: in most cases, Intergalactic absorption systems that are in principle detectable with current or planned X-ray instrumentation will show just a single absorption line, usually the O VII $n=1-2$ resonance line, at an unknown redshift. When assessing the possibility that a given apparent absorption feature is 'real', one has to allow for the number of independent trial redshifts (very roughly given by the width of the wavelength band surveyed, divided by the nominal spectral resolution of the spectrometer), and with a wide band and a high spectral resolution, this tends to dramatically reduce the significance of even fairly impressive apparent absorption dips. For instance, an apparent absorption line detected at a formal ' $3 \sigma$ significance' (or $p=0.0015$ a priori probability for a negative deviation this large to occur due to statistical fluctuation) with Chandra LETGS in the $21.6-23.0 \AA$ band pales to ' $1.7 \sigma$ ' if we assume it is the O VII resonance line in the redshift range $z=0-0.065$; with $N \approx 30$ independent trials, the chances of not seeing a $3 \sigma$ excursion are $(1-p)^{N}=0.956$, or: one will see such a feature one in twenty times if one tries this experiment (we are assuming a Gaussian distribution of fluctuations here). If we allow for confusion with $\mathrm{O}$ VIII Ly $\alpha$ at higher redshift, or even other transitions, the significance is even further reduced. And the larger the number of sources surveyed, the larger 
the probability of false alarm. Clearly, more reliable statistics on intervening X-ray absorbers and detections at higher significance are desired, but the required high-quality data will not be available until the next-generation X-ray facilities such as XEUS and Constellation $X$ are installed (see Paerels et al. 2008-Chap. 19, this issue).

Nevertheless, even with these odds, the above discussed high-ion measurements are important observations to do with the currently available instruments Chandra and XMMNewton. Given the predicted strengths of the absorption lines (e.g., Chen et al. 2003; see also Sect. 5.1), attention has naturally focused on a handful of very bright BL Lac- and similar sources. Below, we discuss the results of the searches. Note that the subject has recently also been reviewed by Bregman (2007).

\subsection{Intervening WHIM Absorbers at Low Redshift}

Early observations of a sample of sources (e.g., S5 0836+710, PKS 2149-306; Fang et al. 2001; PKS 2155-304, Fang et al. 2002) produced no convincing detections. The next attempt at detecting redshifted X-ray $\mathrm{O}$ absorption lines was performed by Mathur et al. (2003) with a dedicated deep observation (470 ks) with the Chandra LETGS of the quasar $\mathrm{H} 1821+643$, which has several confirmed intervening O VI absorbers. No significant X-ray absorption lines were found at the redshifts of the O VI systems, but this was not really surprising in view of the modest signal to noise ratio in the X-ray continuum. Since it requires very bright continua to detect the weak absorption, it is also not surprising that the number of suitable extragalactic sources is severely limited. Nicastro and his colleagues then embarked on a campaign to observe Mrk 421 during its periodic X-ray outbursts, when its X-ray flux rises by an order of magnitude (e.g., Nicastro 2005). The net result of this has been the accumulation of a very deep spectrum with the Chandra LETGS, with a total of more than 7 million continuum counts, in about 1000 resolution elements. Nicastro et al. (2005) have claimed evidence for the detection of two intervening absorption systems in these data, at $z=0.011$ and $z=0.027$. But the spectrum of the same source observed with the XMMNewton RGS does not show these absorption lines (Rasmussen et al. 2007), despite higher signal-to-noise and comparable spectral resolution (Mrk 421 is observed by XMM-Newton for calibration purposes, and by late 2006, more than 1 Ms exposure had been accumulated). Kaastra et al. (2006) have reanalysed the Chandra LETGS data, and find no significant absorption. Other sources, less bright but with larger redshifts, have been observed (see for instance Steenbrugge et al. (2006) for observations of 1ES 1028+511 at $z=0.361$ ), but to date no convincing evidence for intervening absorption has materialised.

Observations have been conducted to try and detect the absorption by intergalactic gas presumably associated with known locations of cosmic overdensity, centred on massive clusters. Fujimoto et al. (2004) attempted to detect absorption in the quasar LBQS 1228+1116, located behind the Virgo cluster. An XMM-Newton RGS spectrum revealed a marginal feature at the (Virgo) redshifted position of O VIII Ly $\alpha$, but only at the $\sim 95 \%$ confidence level. Likewise, Takei et al. (2007) took advantage of the location of X Comae behind the Coma cluster to try and detect absorption from Coma or its surroundings, but no convincing, strong absorption lines were detected in a deep observation with XMM-Newton RGS. The parallel CCD imaging data obtained with EPIC show weak evidence for Ne IX $n=1-2$ line emission at the redshift of Coma, which, if real, would most likely be associated with WHIM gas around the cluster, seen in projection (the cluster virial temperature is too high for Ne IX). In practice, the absence of very bright point sources behind clusters, which makes absorption studies difficult, and the bright foregrounds in emission, will probably make this approach to detecting and characterising the WHIM not much easier than the random line-of-sight searches. 
The conclusion from the search for intergalactic X-ray absorption is that there is no convincing, clear detection for intervening absorption. This is, in retrospect, not that surprising, given the sensitivity of the current X-ray spectrometers, the abundance of suitably bright and sufficiently distant continuum sources, and the predicted properties of the WHIM.

\subsection{The Milky Way Halo and Local Group Gas}

The first positive result of the analysis of bright continuum spectra was the detection of O VII and O VIII $n=1-2$ resonance line absorption at redshift zero. Nicastro et al. (2002) first identified the resonance lines in the Chandra LETGS spectrum of PKS 2155-304 (O VII $n=1-2, n=1-3$, O VIII Ly $\alpha$, Ne IX $n=1-2$ ). Fang et al. (2003) found absorption in the LETGS spectrum of 3C273. Rasmussen et al. (2003) detected resonance absorption in the XMM-Newton RGS spectra of 3C 273, Mrk 421, and PKS 2155-304. Since then, at least O VII $n=1-2$ has been detected in effectively all sufficiently bright continuum sources, both with Chandra and XMM-Newton; a recent compilation appears in Fang et al. (2006). Portions of a deeper spectrum that shows the zero redshift absorption are shown in Fig. 7.

Nicastro et al. (2002) initially interpreted the absorption as arising in an extended intergalactic filament. The argument that drives this interpretation is based on the assumption that $\mathrm{O}$ VI, O VII, and O VIII are all located in a single phase of the absorbing gas. The simultaneous appearance of finite amounts of O VI and O VIII only occurs in photoionised gas, not in
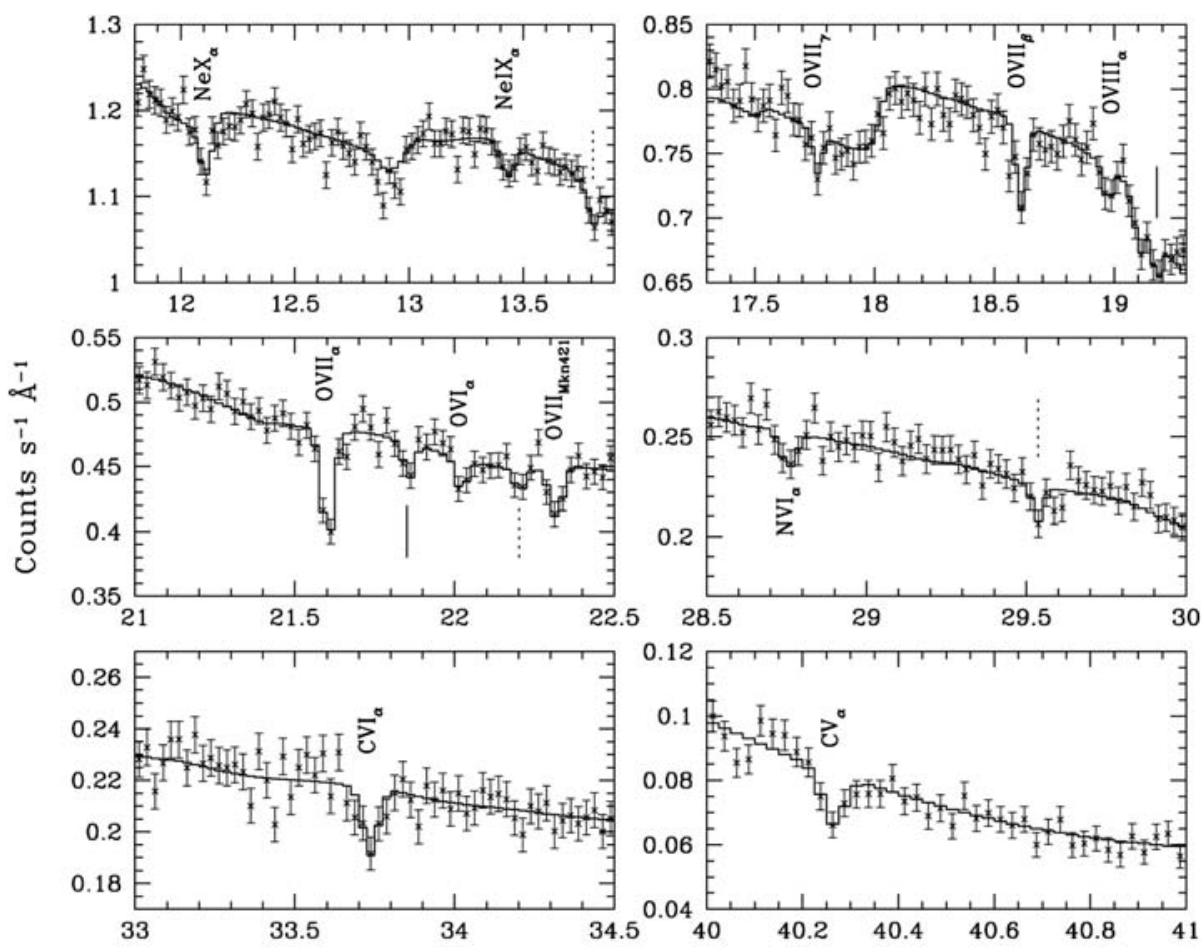

Wavelength $(\AA)$

Fig. 7 Chandra LETGS spectrum of Mrk 421. Crosses are the data, the solid line is a model. The labels identify $z \approx 0$ absorption lines in $\mathrm{Ne}, \mathrm{O}$, and $\mathrm{C}$. The vertical tick marks indicate the locations of possible intergalactic absorption lines. From Williams et al. (2005) 
gas in collisional ionisation equilibrium, and this requires that the gas has very low density (the photoionisation is produced by the local X-ray background radiation field; for a measured ionisation parameter, the known intensity of the ionising field fixes the gas density). The measured ionisation balance then implies a length scale on the order of $l \sim 10\left(Z_{0.1}\right)^{-1}$ Mpc, where $Z_{0.1}$ is the metallicity in units 0.1 Solar. This is a very large length, and even for $0.3 Z_{\odot}$ metallicity, the structure still would not fit in the Local Group (and it is unlikely to have this high a metallicity if it were larger than the Local Group). In fact, the absorption lines should have been marginally resolved in this case, if the structure expands with a fair fraction of the expansion of the Universe.

Rasmussen et al. (2003) constrained the properties of the absorbing gas by dropping the $\mathrm{O}$ VI, and by taking into account the intensity of the diffuse O VII and O VIII line emission as measured by the Wisconsin/Goddard rocket-borne Quantum X-ray Calorimeter (XQC) experiment (McCammon et al. 2002). The cooling timescale of $\mathrm{O}$ VI-bearing gas is much smaller than that of gas with the higher ionisation stages of $\mathrm{O}$, and this justifies the assumption that O VI is located in a different, transient phase of the gas. With only O VII and O VIII, the medium can be denser and more compact, and be in collisional ionisation equilibrium. Treating the measured O VII emission line intensity as an upper limit to the emission from a uniform medium, and constraining the ionisation balance from the measured ratio of $\mathrm{O}$ VII and O VIII column densities in the lines of sight to Mrk 421 and PKS 2155-304, Rasmussen et al. derived an upper limit on the density of the medium of $n_{\mathrm{e}} \lesssim 2 \times 10^{-4} \mathrm{~cm}^{-3}$ and a length scale $l \gtrsim 100 \mathrm{kpc}$.

Bregman (2007) favours a different solution, with a lower electron temperature and hence a higher O VII ion fraction. If one assumes Solar abundance and sets the O VII fraction to 0.5 , the characteristic density becomes $n_{e} \sim 10^{-3} \mathrm{~cm}^{-3}$, and the length scale $(l \sim 20 \mathrm{kpc})$ suggests a hot Galactic halo, rather than a Local Group intragroup medium.

Arguments for both type of solution (a small compact halo and a more tenuous Local Group medium) can be given. The most direct of these is a measurement of the O VII line absorption towards the LMC by Wang et al. (2005) in the spectrum of the X-ray binary LMC $\mathrm{X}-3$, which indicates that a major fraction of the O VII column in that direction is in fact in front of the LMC. Bregman (2007) points out that the distribution of column densities of highly ionised $\mathrm{O}$ on the sky is not strongly correlated with the likely projected mass distribution of the Local Group, and that the measured velocity centroid of the absorption lines appears characteristic of Milky Way gas, rather than Local Group gas. On the other hand, a direct measurement of the Doppler broadening of the OVII gas, from the curve of growth of the $n=1-2$ and $n=1-3$ absorption lines in the spectrum of Mrk 421 and PKS 2155-304 (Williams et al. 2005, 2007), indicates an ion temperature of $T_{\mathrm{i}} \approx 10^{6.0-6.3} \mathrm{~K}$ (Mrk 421) and $T_{i} \approx 10^{6.2-6.4} \mathrm{~K}$ (PKS 2155-304), and these values favour the low-density, Local Group solution. Regardless, the prospect of directly observing hot gas expelled from the Galactic disk, or measuring the virial temperature of the Galaxy and/or the Local Group is exciting enough to warrant further attention to redshift zero absorption and emission.

Finally, the spectrum of Mrk 421 shows the expected $z=0$ innershell O VI absorption, at $22.019 \AA$, both with Chandra and with XMM-Newton. There has been some confusion regarding an apparent discrepancy between the O VI column densities derived from the FUV and from the X-ray absorption lines, in the sense that the X-ray column appeared to be significantly larger than the FUV column (Williams et al. 2005). Proposed physical explanations for this effect involve a depletion of the lower level of the FUV transitions $\left(1 s^{2} 2 s\right)$ in favour of (at least) $1 s^{2} 2 p$, which weakens the $\lambda \lambda 1032,1038 \AA$ absorption but does not affect the $1 s-2 p \mathrm{X}$-ray absorption. However, it requires very high densities to maintain a finite excited state population, and, more directly, the measured wavelength of the X-ray line 
is actually not consistent with the wavelength calculated for $1 s-2 p$ in excited O VI, off by about $0.03-0.05 \AA$, on the order of a full resolution element of both the Chandra LETGS and the XMM-Newton RGS (Raassen 2007, private communication). The conclusion is that the discrepancy is due to an authentic statistical fluctuation in the X-ray spectrum-or, more ironically, to the presence of a weak, slightly redshifted O VII absorption line.

\section{Additional Aspects}

\subsection{Results from Numerical Simulations}

Cosmological simulations not only have been used to investigate the large-scale distribution and physical state of the warm-hot intergalactic medium, they also have been applied to predict statistical properties of high-ion absorption systems that can be readily compared with the UV and X-ray measurements (e.g., Cen et al. 2001; Fang and Bryan 2001; Chen et al. 2003; Furlanetto et al. 2005; Tumlinson and Fang 2005; Cen and Fang 2006). Usually, a large number of artificial spectra along random sight-lines through the simulated volume are generated. Sometimes, instrumental properties of existing spectrographs and noise characteristics are modelled, too (e.g., Fangano et al. 2007). The most important quantities derived from artificial spectra that can be compared with observational data are the cumulative and differential number densities $(\mathrm{d} N / \mathrm{d} z)$ of $\mathrm{O}$ VI, O VII, O VIII systems as a function of the absorption equivalent width. An example for this is shown in Fig. 8. Generally, there is a good match between the simulations and observations for the overall shape of the $\mathrm{d} N / \mathrm{d} z$ distribution (see also Sect. 3.2), but mild discrepancies exist at either low or high equivalent widths, depending on what simulation is used (see, e.g., Tripp et al. 2007). For the interpretation of such discrepancies it is important to keep in mind that the different simulations are based on different physical models for the gas, e.g., some simulations include galaxy feedback models, galactic wind models, non-equilibrium ionisation conditions, etc., others do not. For more information on numerical simulations of the WHIM see Bertone et al. (2008-Chap. 14, this issue).

WHIM simulations also have been used to investigate the frequency and nature of BLAs at low redshift (Richter et al. 2006b). As the simulations suggest, BLAs indeed host a substantial fraction of the baryons at $z=0$. From the artificial UV spectra generated from their simulation Richter et al. derive a number of BLAs per unit redshift of $(\mathrm{d} N / \mathrm{d} z)_{\mathrm{BLA}} \approx 38$ for

Fig. 8 The differential number of intervening oxygen high-ion (O VI, O VII,O VIII) absorbers in the WHIM in a cosmological simulation is plotted against the equivalent width of the absorption (for details see Cen and Fang 2006). While for O VII and $\mathrm{O}$ VIII no significant observational results are available to be compared with the simulated spectra (see Sect. 4.2), the predicted frequency of $\mathrm{O}$ VI absorbers is in good agreement with the observations (Sect. 3.2). Adapted from Cen and Fang (2006)

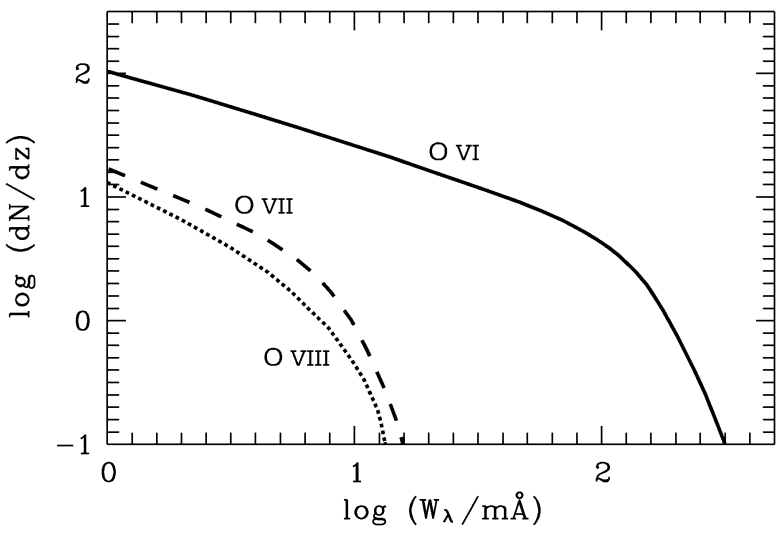



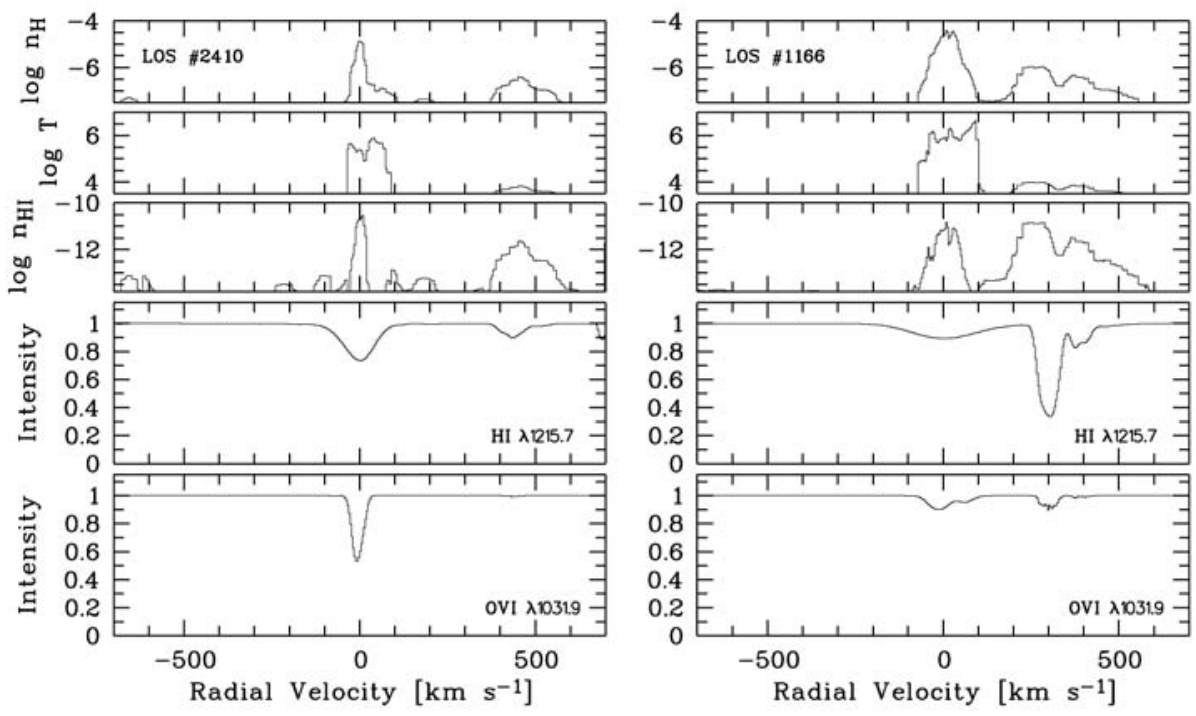

Fig. 9 Two examples for BLA absorbers from the WHIM in a cosmological simulation are shown. The panels show the logarithmic total hydrogen volume density, gas temperature, neutral hydrogen volume density, and normalised intensity for $\mathrm{H} \mathrm{I} \mathrm{Ly} \alpha$ and $\mathrm{O}$ VI $\lambda 1031.9$ absorption as a function of the radial velocity along each sightline. From Richter et al. (2006b)

H I absorbers with $\log \left(N\left(\mathrm{~cm}^{-2}\right) / b\left(\mathrm{~km} \mathrm{~s}^{-1}\right)\right) \geq 10.7, b \geq 40 \mathrm{~km} \mathrm{~s}^{-1}$, and total hydrogen column densities $N(\mathrm{H} \mathrm{II}) \leq 10^{20.5} \mathrm{~cm}^{-2}$. The baryon content of these systems is $\sim 25$ percent of the total baryon budget in the simulation. These results are roughly in line with the observations if partial photoionisation of BLAs is taken into account (Richter et al. 2006a; Lehner et al. 2007). From the simulation further follows that BLAs predominantly trace shock-heated collisionally ionised WHIM gas at temperatures $\log T \approx 4.4-6.2$. Yet, about 30 percent of the BLAs in the simulation originate in the photoionised Ly $\alpha$ forest $(\log T<4.3)$ and their large line widths are determined by non-thermal broadening effects such as unresolved velocity structure and macroscopic turbulence. Figure 9 shows two examples of the velocity profiles of BLAs generated from simulations presented in Richter et al. (2006b).

The results from the analysis of artificially generated UV spectra underline that the comparison between WHIM simulations and quasar absorption line studies indeed are quite important for improving both the physical models in cosmological simulations and the strategies for future observations of the warm-hot intergalactic gas.

\subsection{WHIM Absorbers at High Redshift}

Although this chapter concentrates on the properties of WHIM absorbers at low redshift (as visible in UV and X-ray absorption) a few words about high-ion absorption at high redshifts $(z>2)$ shall be given at this point. At redshifts $z>2$, by far most of the baryons are residing in the photoionised intergalactic medium that gives rise to the Ly $\alpha$ forest. At this early epoch of the Universe, baryons situated in galaxies and in warm-hot intergalactic gas created by large-scale structure formation contribute together with only $<15$ percent to the total baryon content of the Universe. Despite the relative unimportant role of the WHIM at high $z, \mathrm{O}$ VI absorbers are commonly found in optical spectra of high-redshift quasars (e.g., Bergeron et al. 2002; Carswell et al. 2002; Simcoe et al. 2004). The observation of intervening O VI 
absorbers at high redshift is much easier than in the local Universe, since the absorption features are redshifted into the optical regime and thus are easily accessible with groundbased observatories. However, blending problems with the numerous $\mathrm{H} \mathrm{I} \mathrm{Ly} \alpha$ forest lines at high $z$ are much more severe than for low-redshift sightlines. Because of the higher intensity of the metagalactic UV background at high redshift it is expected that many of the O VI systems in the early Universe are photoionised. Collisional ionisation of O vi yet may be important for high-redshift absorbers that originate in galactic winds (see, e.g., Fangano et al. 2007). While for low redshifts the population of O VI absorbers is important for the search of the "mission baryons" that are locked in the WHIM phase in the local Universe, O VI absorbers at high redshift are believed to represent a solution for the problem of the "missing metals" in the early epochs of structure formation. This problem arises from the facts that at high redshift an IGM metallicity of $\sim 0.04$ is expected from the star-formation activity of Lyman-Break Galaxies (LBGs), while observations of intervening C IV absorption systems suggest an IGM abundance of only 0.001 solar (Songaila 2001; Scannapieco et al. 2006), thus more than one order of magnitude too low. Possibly, most of the missing metals at high $z$ are hidden in highly-ionised hot gaseous halos that surround the star-forming galaxies (e.g., Ferrara et al. 2005) and thus should be detectable only with high ions such as O vi rather than with intermediate ions such as CIV. Using the UVES spectrograph installed on the Very Large Telescope (VLT) Bergeron and Herbert-Fort (2005) have studied the properties of high-redshift O VI absorbers along ten QSO sightlines and have found possible evidence for such a scenario. Additional studies are required to investigate the nature of high- $z \mathrm{O}$ VI systems and their relation to galactic structures in more detail. However, from the existing measurements clearly follows that the study of high-ion absorbers at large redshifts is of great importance to our understanding of the formation and evolution of galactic structures at high $z$ and the transport of metals into the IGM.

\section{Concluding Remarks}

The analysis of absorption features from high ions of heavy elements and neutral hydrogen currently represents the best method to study baryon content, physical properties, and distribution of the warm-hot intergalactic gas in large-scale filaments at low and high redshift. However, the interpretation of these spectral signatures in terms of WHIM baryon content and origin still is afflicted with rather large systematic uncertainties due to the limited data quality and the often poorly known physical conditions in WHIM absorbers (e.g., ionisation conditions, metal content, etc.). Future instruments in the UV (e.g., COS) and in the X-ray band (e.g., XEUS, Constellation X) hold the prospect of providing large amounts of new data on the WHIM with good signal-to-noise ratios and substantially improved absorber statistics. These missions therefore will be of great importance to improve our understanding of this important intergalactic gas phase.

Acknowledgements The authors thank ISSI (Bern) for support of the team "Non-virialised X-ray components in clusters of galaxies". SRON is supported financially by NWO, the Netherlands Organisation for Scientific Research.

\section{References}

M. Asplund, N. Grevesse, A.J. Sauval, C. Allende Prieto, D. Kiselman, Astron. Astrophys. 417, 751 (2004)

J. Bergeron, S. Herbert-Fort, in Probing Galaxies through Quasar Absorption Lines, ed. by P.R. Williams, C.-G. Shu, B. Menard. IAU Coll. 199 (Cambr. Univ. Press, 2005), p. 265 
J. Bergeron, B. Aracil, P. Petitjean, C. Pichon, Astron. Astrophys. 396, L11 (2002)

S. Bertone, J. Schaye, K. Dolag, Space Sci. Rev. (2008). doi:10.1007/s11214-008-9318-3

D.V. Bowen, M. Pettini, J.C. Blades, Astrophys. J. 580, 169 (2002)

J.N. Bregman, Annu. Rev. Astron. Astrophys. 45, 221 (2007)

A.C. Brinkman, C.J.T. Gunsing, J.S. Kaastra et al., Astrophys. J. 530, L111 (2000)

A.M. Bykov, F.B.S. Paerels, V. Petrosian, Space Sci. Rev. (2008). doi:10.1007/s11214-008-9309-4

C.R. Canizares, J. Kruper, Astrophys. J. 278, L99 (1984)

C.R. Canizares, G.W. Clark, T.H. Markert et al., Astrophys. J. 234, L33 (1979)

C.R. Canizares, J.E. Davis, D. Dewey et al., Publ. Astron. Soc. Pac. 117, 1144 (2005)

B. Carswell, J. Schaye, T.-S. Kim, Astrophys. J. 578, 43 (2002)

R. Cen, T. Fang, Astrophys. J. 650, 573 (2006)

R. Cen, J. Ostriker, Astrophys. J. 514, 1 (1999)

R. Cen, T.M. Tripp, J.P. Ostriker, E.B. Jenkins, Astrophys. J. 559, L5 (2001)

H.-W. Chen, J.X. Prochaska, Astrophys. J. 543, L9 (2000)

X. Chen, D. Weinberg, N. Katz, R. Davé, Astrophys. J. 594, 42 (2003)

C.W. Danforth, J.M. Shull, Astrophys. J. 624, 555 (2005)

R. Davé, R. Cen, J. Ostriker et al., Astrophys. J. 552, 473 (2001)

J.W.A. den Herder, A.C. Brinkman, S.M. Kahn et al., Astron. Astrophys. 365, L7 (2001)

G.W. Drake, Can. J. Phys. 66, 586 (1988)

T. Fang, G.L. Bryan, Astrophys. J. 561, L31 (2001)

T. Fang, H.L. Marshall, G.L. Bryan, C.R. Canizares, Astrophys. J. 555, 356 (2001)

T. Fang, H.L. Marshall, J.C. Lee, D.S. Davis, C.R. Canizares, Astrophys. J. 572, L127 (2002)

T. Fang, K.R. Sembach, C.R. Canizares, Astrophys. J. 586, L49 (2003)

T. Fang, R.A.C. Croft, W.T. Sanders et al., Astrophys. J. 623, 612 (2005)

T. Fang, C.F. McKee, C.R. Canizares, M. Wolfire, Astrophys. J. 644, 174 (2006)

A.P.M. Fangano, A. Ferrara, P. Richter, Mon. Not. R. Astron. Soc. 381, 469 (2007)

A. Ferrara, E. Scannapieco, J. Bergeron, Astrophys. J. 634, L37 (2005)

A.J. Fox, B.D. Savage, B.P. Wakker et al., Astrophys. J. 602, 738 (2004)

R. Fujimoto, Y. Takei, T. Tamura et al., Publ. Astron. Soc. Jpn. 56, L29 (2004)

S.R. Furlanetto, L.A. Phillips, M. Kamionkowski, Mon. Not. R. Astron. Soc. 359, 295 (2005)

F. Haardt, P. Madau, Astrophys. J. 461, 20 (1996)

W.R. Johnson, G. Soff, Atom. Data Nucl. Data Tables 33, 405 (1985)

J.S. Kaastra, N. Werner, J.W.A. den Herder et al., Astrophys. J. 652, 189 (2006)

J.S. Kaastra, F.B.S. Paerels, F. Durret, S. Schindler, P. Richter, Space Sci. Rev. (2008). doi:10.1007/s11214008-9310-y

H. Kawahara, K. Yoshikawa, S. Sasaki et al., Publ. Astron. Soc. Jpn. 58, 657 (2006)

R.A. Kimble, B.E. Woodgate, C.W. Bowers et al., Astrophys. J. 492, L83 (1998)

N. Lehner, B.D. Savage, P. Richter et al., Astrophys. J. 658, 680 (2007)

R. Lynds, Astrophys. J. 164, L73 (1971)

S. Mathur, D.H. Weinberg, X. Chen, Astrophys. J. 582, 82 (2003)

D. McCammon, R. Almy, E. Apodaca et al., Astrophys. J. 576, 188 (2002)

H.W. Moos, W.C. Cash, L.L. Cowie et al., Astrophys. J. 538, L1 (2000)

F. Nicastro, in Maps of the Cosmos, ed. by M. Colless, L. Staveley-Smith, R. Stathakis. IAU Symp. 216 (Astr. Soc. Pac., 2005), p. 297. astro-ph/0311162

F. Nicastro, A. Zezas, J. Drake et al., Astrophys. J. 573, 157 (2002)

F. Nicastro, A. Zezas, M. Elvis et al., Nature 421, 719 (2003)

F. Nicastro, S. Mathur, M. Elvis et al., Astrophys. J. 629, 700 (2005)

W.R. Oegerle, T.M. Tripp, K.R. Sembach et al., Astrophys. J. 538, L23 (2000)

F.B.S. Paerels, J.S. Kaastra, T. Ohashi et al., Space Sci. Rev. (2008). doi:10.1007/s11214-008-9323-6

M. Pettini, S.L. Ellison, J. Bergeron, P. Petitjean, Astron. Astrophys. 391, 21 (2002)

N. Prause, D. Reimers, C. Fechner, E. Janknecht, Astron. Astrophys. 470, 67 (2007)

A.P. Rasmussen, S.M. Kahn, F.B.S. Paerels, in The IGM/Galaxy Connection: The Distribution of Baryons at $z=0$, ed. by J.L. Rosenberg, M.E. Putman. ASSL Conf. Proc., vol. 281 (Kluwer, Dordrecht, 2003), p. 109. astro-ph/0301183

A.P. Rasmussen, S.M. Kahn, F. Paerels et al., Astrophys. J. 656, 129 (2007)

P. Richter, Rev. Mod. Astron. 19, 31 (2006c)

P. Richter, B.D. Savage, T.M. Tripp, K.R. Sembach, Astrophys. J. Suppl. Ser. 153, 165 (2004)

P. Richter, B.D. Savage, K.R. Sembach, T.M. Tripp, Astron. Astrophys. 445, 827 (2006a)

P. Richter, T. Fang, G.L. Bryan, Astron. Astrophys. 451, 767 (2006b)

D.J. Sahnow, H.W. Moos, T.B. Ake et al., Astrophys. J. 538, L7 (2000)

B.D. Savage, K.R. Sembach, E.B. Jenkins et al., Astrophys. J. 538, L27 (2000) 
B.D. Savage, K.R. Sembach, T.M. Tripp, P. Richter, Astrophys. J. 564, 631 (2002)

B.D. Savage, N. Lehner, B.P. Wakker, T.M. Sembach, K.R. Tripp, Astrophys. J. 626, 776 (2005)

B.D. Savage, K.R. Sembach, B.P. Wakker et al., Astrophys. J. Suppl. Ser. 146, 125 (2003)

E. Scannapieco, C. Pichon, B. Aracil et al., Mon. Not. R. Astron. Soc. 365, 615 (2006)

M. Schmidt, P. Beiersdorfer, H. Chen et al., Astrophys. J. 604, 562 (2004)

K.R. Sembach, B.P. Wakker, B.D. Savage et al., Astrophys. J. Suppl. Ser. 146, 165 (2003)

K.R. Sembach, T.M. Tripp, B.D. Savage, P. Richter, Astrophys. J. Suppl. Ser. 155, 351 (2004)

J.M. Shull, S.V. Penton, J.T. Stocke et al., Astron. J. 116, 2094 (1998)

R.A. Simcoe, W.L.W. Sargent, M. Rauch, Astrophys. J. 606, 92 (2004)

A. Songaila, Astrophys. J. 561, L153 (2001)

K. Steenbrugge, F. Nicastro, M. Elvis, in The X-ray Universe 2005, ed. by A. Wilson. ESA SP-604, 2006, p. 751. astro-ph/0511234

J.T. Stocke, J.M. Shull, S. Penton, M. Donahue, C. Carilli, Astrophys. J. 451, 24 (1995)

J.T. Stocke, S.V. Penton, C.W. Danforth et al., Astrophys. J. 641, 217 (2006)

R.S. Sutherland, M.A. Dopita, Astrophys. J. Suppl. Ser. 88, 253 (1993)

Y. Takei, J.P. Henry, A. Finoguenov et al., Astrophys. J. 655, 831 (2007)

T.M. Tripp, L. Lu, B.D. Savage, Astrophys. J. 508, 200 (1998)

T.M. Tripp, B.D. Savage, E.B. Jenkins, Astrophys. J. 534, L1 (2000)

T.M. Tripp, M.L. Giroux, J.T. Stocke, J. Tumlinson, W.R. Oegerle, Astrophys. J. 563, 724 (2001)

T.M. Tripp, K.R. Sembach, D.V. Bowen et al., Astrophys. J. Suppl. Ser. (2007, in press). astro-ph/07061214

J. Tumlinson, T. Fang, Astrophys. J. 623, L100 (2005)

P. Valageas, R. Schaeffer, J. Silk, Astron. Astrophys. 388, 741 (2002)

D.A. Verner, E.M. Verner, G.J. Ferland, Atom. Data Nucl. Data Tables 64, 1 (1996)

B.P. Wakker, B.D. Savage, K.R. Sembach et al., Astrophys. J. Suppl. Ser. 146, 1 (2003)

Q.D. Wang, Y. Yao, T.M. Tripp et al., Astrophys. J. 635, 386 (2005)

R.J. Williams, S. Mathur, F. Nicastro et al., Astrophys. J. 631, 856 (2005)

R.J. Williams, S. Mathur, F. Nicastro, M. Elvis, Astrophys. J. 665, 247 (2007)

B.E. Woodgate, Publ. Astron. Soc. Pac. 110, 1183 (1998) 